\title{
An activated-platelet-sensitive nanocarrier enables targeted delivery of tissue plasminogen activator for effective thrombolytic therapy
}

Yu Huang ${ }^{\mathrm{a}}$, Li Yu ${ }^{\mathrm{a}}$, Jie Ren ${ }^{\mathrm{a}}$, Boram Gu ${ }^{\mathrm{a}}$, Colin Longstaff ${ }^{\mathrm{b}}$, Alun D. Hughes ${ }^{\mathrm{c}, \mathrm{d}}$, Simon A. Thom ${ }^{\mathrm{e}}$, Xiao Yun $\mathrm{Xu}^{\mathrm{a}}$, and Rongjun Chen*, a

${ }^{a}$ Department of Chemical Engineering, Imperial College London, South Kensington Campus, London, United Kingdom

b Biotherapeutics Section, National Institute for Biological Standards and Control, South Mimms, Herts, United Kingdom

${ }^{\mathrm{c}}$ Institute of Cardiovascular Science, University College London, London, United Kingdom

${ }^{\mathrm{d}}$ MRC Unit for Lifelong Health and Ageing at University College London, London, United Kingdom

e National Heart \& Lung Institute, Imperial College London, Hammersmith Campus, London, United Kingdom

* Corresponding author: Dr Rongjun Chen.

Tel: +44 (0)20 75942070 .

Fax: +44 (0)20 75945638.

E-mail: rongjun.chen@imperial.ac.uk. 


\section{ABSTRACT}

It remains a major challenge to develop a selective and effective fibrinolytic system for thrombolysis with minimal undesirable side effects. Herein, we report a multifunctional liposomal system $(164.6 \pm 5.3 \mathrm{~nm}$ in diameter $)$ for selective thrombolysis through targeted delivery and controlled release of tissue plasminogen activator (tPA) at the thrombus site. The tPA-loaded liposomes were PEGylated to improve their stability, and surface coated with a conformationally-constrained, cyclic arginine-glycine-aspartic acid (cRGD) to enable highly selective binding to activated platelets. The in vitro drug release profiles at $37{ }^{\circ} \mathrm{C}$ showed that over $90 \%$ of tPA was released through liposomal membrane destabilization involving membrane fusion upon incubation with activated platelets within $1 \mathrm{~h}$, whereas passive release of the encapsulated tPA in $\mathrm{pH}$ 7.4 PBS buffer was $10 \%$ after $6 \mathrm{~h}$. The release of tPA could be readily manipulated by changing the concentration of activated platelets. The presence of activated platelets enabled the tPA-loaded, cRGD-coated, PEGylated liposomes to induce efficient fibrin clot lysis in a fibrin-agar plate model and the encapsulated tPA retained $97.4 \pm$ $1.7 \%$ of fibrinolytic activity as compared with that of native tPA. Furthermore, almost complete blood clot lysis was achieved in $75 \mathrm{~min}$, showing considerably higher and quicker thrombolytic activity compared to the tPA-loaded liposomes without cRGD labelling. These results suggest that the nano-sized, activated-platelet-sensitive, multifunctional liposomes could facilitate selective delivery and effective release of tPA at the site of thrombus, thus achieving efficient clot dissolution whilst minimising undesirable side effects.

Keywords: Activated platelet; liposome; tissue plasminogen activator; GPIIb-IIIa integrin; targeted delivery; controlled release mechanism; thrombolysis 


\section{Introduction}

The formation of a blood clot can cause vessel obstruction or occlusion, leading to catastrophic events such as ischemic stroke, acute myocardial infarction and pulmonary embolism $[1,2]$. Timely removal of occlusive arterial clots to rapidly re-establish blood flow is critical to treat these diseases [3]. One of the currently available clinical strategies for clot lysis is intravenous infusion of a fibrinolytic agent such as urokinase, streptokinase and tissue plasminogen activators (tPA), which can convert plasminogen to plasmin and thus trigger fibrin lysis to break up clots [4-6]. Unfortunately, these thrombolytic agents have short circulation lives (e.g. only 2 6 min of half-life for tPA) [7-9]. Thus, large drug doses are required for effective thrombolysis. However, excessive administration and systemic distribution of these fibrinolytic drugs are detrimental because they may impair normal hemostatic capabilities and lead to bleeding complications [10].

Although many efforts have been made to improve the stability of thrombolytics for prolonged blood circulation by PEGylation or encapsulation [11-18], achieving effective drug accumulation at the clot-site to enable targeted thrombolysis still remains a major challenge [19]. Korin et al. reported poly(lactic-co-glycolic acid)-based, shear-active nanotherapeutics, by taking advantage of the high shear stress caused by arterial narrowing for targeted delivery of tPA to obstructed blood vessels [20]. Another targeted delivery system has been developed by utilizing magnetically-activated nanomotors to improve the transport of tPA at the blood clot surface for more effective local ischemic stroke therapy [21]. Nevertheless, the dependence on luminal high shear stress or an externally rotating magnetic nanomotor makes it difficult to implement such systems in a general clinical setting [22, 23]. Alternatively, 
thrombus-activated delivery strategies in response to factors present in the characteristic microenvironment of a thrombus site, such as thrombin [24, 25], fibrin network [26, 27], collagen [28], glycoprotein [29] and P-selectin [30-32], provide a new perspective for the design of clot-specific therapeutics. Among these, upregulation of GPIIb-IIIa $\left(\alpha_{\text {IIb }} \beta_{3}\right)$ integrin on activated platelets has been described as a significant hallmark of thrombus, because $\alpha_{\text {IIb }} \beta_{3}$ integrins are inactive on resting platelets but are abundantly expressed and activated on the platelet surface in the event of thrombus formation [33-38]. Therefore, it is attractive to develop delivery systems that are sensitive to activated platelets and bind $\alpha_{\mathrm{IIb}} \beta_{3}$ integrins, thus enabling targeted delivery and controlled release of thrombolytic agents for selective and effective thrombolysis.

Several nanocarriers have been reported to enhance the targeted delivery of tPA (the most widely used fibrinolytic agent) to a thrombus in vitro and in vivo using peptide sequences of fibrinogen, such as arginine-glycine-aspartic acid (RGD) motifs located in each of its two A $\alpha$ chains and the sequence (CQQHHLGGAKQAGDV) located within its $\gamma$ chains that selectively bind to $\alpha_{\mathrm{IIb}} \beta_{3}$ integrins on the surface of activated platelets [39-41]. However, drug release mechanisms at the clot site remain unknown and the role of activated platelets in the controlled release process is not understood. Herein, we report an activated-platelet-sensitive nanocarrier for selective thrombolysis through targeted delivery and controlled release of tPA to the blood clot. In this nanothrombolytic system, tPA was encapsulated in a PEGylated liposome with the potential for improved stability. The tPA-loaded, PEGylated liposome was surface coated with a conformationally-constrained, cyclic RGD (cRGD) peptide which has been reported to have a much higher specificity and affinity for $\alpha_{\mathrm{IIb}} \beta_{3}$ integrins compared to linear RGD structures 
[16]. The controlled release of tPA mediated by the selective interaction of the multifunctional liposome with activated platelets was demonstrated and the release mechanism was elucidated. The efficient and selective lysis of fibrin and blood clots suggest that the multifunctional liposomal system is a promising candidate for targeted thrombolytic therapy with reduced undesirable side effects.

\section{Materials and methods}

\subsection{Materials}

L- $\alpha$-phosphatidylcholine from egg yolk (EPC), cholesterol, 1-(3-dimethylaminopropyl)-3-ethyl carbodiimide hydrochloride (EDC), N-hydroxy succinimide (NHS), 4-dimethylaminopyridine (DMAP), Dulbecco's phosphate-buffered saline (PBS), N-(2-hydroxyethyl) piperazine-N'-(2-ethanesulfonic acid) (HEPES), calcium chloride $\left(\mathrm{CaCl}_{2}\right)$, sodium chloride $(\mathrm{NaCl})$, fluorescein isothiocyanate (FITC), cyclic RGD (cyclo(Arg-Gly-Asp-D-Phe-Val)), Triton X-100, thrombin, plasminogen, fibrinogen, tris (hydroxymethyl) aminomethane, collagen, the bicinchoninic acid (BCA) protein assay kit and, tPA chromogenic activity assay kit S-2251, calcein, eptifibatide, L- $\alpha$-phosphatidylethanolamine-N-(7-nitro-2-1, 3-benzoxadiazol-4-yl) (ammonium salt) (NBD-PE), and L- $\alpha$-phosphatidylethanolamine-N-(lissamine rhodamine B sulfonyl) (ammonium salt) (Rhod-PE) were purchased from Sigma-Aldrich (Dorset, UK). Centrifugal concentrators were purchased from Fisher Scientific (Loughborough, UK). tPA (alteplase) was a product of Boehringer Ingelheim (Germany). Whole sheep blood in acid citrate dextrose (ACD) was obtained from TCS Biosciences Ltd (Buckingham, UK). The extruder set, 1, 2-distearoyl-sn-glycero-3-phosphoethanolamine-N-[amino(polyethylene glycol)-2000] 
(DSPE-PEG-NH $\mathrm{N}_{2}$ ), polycarbonate membranes and filter supports were purchased from Avanti Polar Lipids Inc. (Alabaster, USA). Chloroform $\left(\mathrm{CHCl}_{3}\right)$, deuterated chloroform $\left(\mathrm{CDCl}_{3}\right)$, dimethyl sulfoxide (DMSO), deuterated dimethyl sulfoxide (DMSO- $d_{6}$ ), absolute ethyl alcohol (EA), acetone, hydrochloric acid, sodium hydroxide and other chemicals were obtained from VWR (Lutterworth, UK).

\subsection{Preparation of FITC-labelled tPA}

$1 \mathrm{~mL}$ of FITC solution in DMSO $\left(1.0 \mathrm{mg} \mathrm{mL}^{-1}\right)$ was added dropwise into $2 \mathrm{~mL}$ of tPA solution in PBS buffer at $\mathrm{pH} 8.0\left(1.0 \mathrm{mg} \mathrm{mL}^{-1}\right)$ and stirred at $4{ }^{\circ} \mathrm{C}$ in the dark overnight. The mixture was dialyzed against pH 7.4 PBS solution for $24 \mathrm{~h}(\mathrm{MWCO}=3500 \mathrm{Da})$ and the purified product was stored at $4{ }^{\circ} \mathrm{C}$ before use.

\section{3. tPA chromogenic activity assay}

The tPA activity was determined by using chromogenic substrate of S-2251. Firstly, 96-well cell culture plates were coated with $5 \%$ bovine serum albumin (BSA) solution to inhibit non-specific absorption, followed by washing with PBS buffer at $\mathrm{pH} 7.4$ for three times and then drying at room temperature. $1.3 \mathrm{mM}$ substrate $\mathrm{S}-2251$ in $\mathrm{H}_{2} \mathrm{O}$ and $0.24 \mu \mathrm{M}$ plasminogen in Tris- $\mathrm{HCl}$ buffer ( $\mathrm{pH} 7.4,0.05 \mathrm{M}$ Tris- $\mathrm{HCl}$ and $0.05 \mathrm{M} \mathrm{NaCl})$ were mixed at a volume ratio of $1: 3$, and $100 \mu \mathrm{L}$ of the plasmin substrate solution was placed into each well. The tPA solution was then added into the well with shaking for $5 \mathrm{~min}$. The absorbance of the mixture at $405 \mathrm{~nm}$ was measured by a GloMax-Multi Microplate Multimode Reader (Promega, USA), and the tPA activity was determined according to the calibration curve obtained by using the standard solutions of free tPA. 


\subsection{Synthesis and characterization of cRGD-modified lipid}

DSPE-PEG- $\mathrm{NH}_{2}$ was grafted to the free carboxyl group of the cyclic RGD by amidation reaction. Equimolar quantities of DSPE-PEG-NH $\mathrm{NH}_{2}$ and the cRGD peptide were added together to react in dried DMSO in the presence of excess NHS and equimolar quantity of EDC. The solution was stirred at $35{ }^{\circ} \mathrm{C}$ for $36 \mathrm{~h}$. Synthesized DSPE-PEG-cRGD was purified by dialysis against distilled water for 2 days. Final product DSPE-PEG-cRGD was characterized by ${ }^{1} \mathrm{H}$ NMR (Bruker, Germany) in DMSO- $d_{6}$ (Figure S1) at room temperature.

\subsection{Preparation of tPA-loaded, cRGD-coated, PEGylated liposomes}

EPC, DSPE-PEG-cRGD and cholesterol at the required ratios were dissolved in a binary organic solvent system $\left(\mathrm{CHCl}_{3}: \mathrm{EA}=5: 1, \mathrm{v}: \mathrm{v}\right)$. A lipid film was formed after removal of the organic solvents by rotary evaporation for $6 \mathrm{~h}$, and then hydrated with PBS buffer at $\mathrm{pH} 7.4$ and $40{ }^{\circ} \mathrm{C}$. After $1 \mathrm{~h}$, the resulting liposomes were repeatedly extruded for 31 times through $200-\mathrm{nm}$ polycarbonate membranes and filter supports. The tPA-loaded liposomes were prepared by hydrating the lipid film with $2 \mathrm{mg} \mathrm{mL}^{-1}$ tPA solution in PBS buffer at $\mathrm{pH} 7.4$ (Figure S2). The unencapsulated tPA was removed by ultracentrifugation (Eppendorf, UK) for $45 \mathrm{~min}$.

\subsection{Characterization of tPA-loaded multifunctional liposomes}

The hydrodynamic size and polydispersity index (PDI) of the liposomal systems were measured at $25{ }^{\circ} \mathrm{C}$ by dynamic light scattering (DLS) using a Zetasizer Nano S (Malvern, UK), and their zeta potential was measured using a ZetaPALS Zeta Potential Analyser (Brookhaven, USA). Liposomal morphology was evaluated by transmission electron microscopy (TEM) using a JEOL JEM-2100F instrument (Japan). TEM samples were prepared by dropping the 
liposomal dispersion in $\mathrm{pH} 7.4 \mathrm{PBS}$ buffer on formvar carbon-coated copper grids. The stability of the tPA-loaded liposomes was measured by monitoring the size change and tPA leakage during storage at $4^{\circ} \mathrm{C}$. The tPA encapsulation efficiency was assessed by determining the tPA activity after solubilizing the liposomal membrane with $0.1 \%$ Triton $\mathrm{X}-100$. Specifically, the tPA-loaded multifunctional liposomes were first purified by ultracentrifuge at $200,000 \times \mathrm{g}$ for $1 \mathrm{~h}$. Then the pellets were re-suspended with PBS buffer at $\mathrm{pH} 7.4$, followed by liposomal lysis with Triton X-100 (1:1, v:v) at $37{ }^{\circ} \mathrm{C}$ for $15 \mathrm{~min}$. The assay of tPA activity was carried out to record the amounts of added tPA and incorporated tPA, respectively. The encapsulation efficiency (EE) was then calculated by the following equation:

$$
\mathrm{EE}(\%)=\frac{m_{l}}{m_{t}} \times 100
$$

where $m_{l}$ is the weight of tPA loaded and $m_{t}$ is the total weight of tPA initially in the loading solution.

\subsection{Isolation of platelets}

Platelets were isolated from whole sheep blood with anti-coagulant ACD by differential centrifugation. As shown in Figure S3, the blood was placed into a $1 \mathrm{~mL}$ tube and centrifuged at $200 \times \mathrm{g}$ for $15 \mathrm{~min}$. Platelet-rich plasma (PRP) was obtained by removing red blood cells, and platelets were then collected by centrifugation of PRP at $800 \times \mathrm{g}$ for $15 \mathrm{~min}$. Platelets were re-suspended in PBS buffer with the presence of anti-coagulant ACD at $\mathrm{pH} 7.4$ and counted for experimental use.

\subsection{Binding affinity with activated platelets}


To evaluate the potential of the multifunctional liposomes for targeted thrombolysis, their selective binding to activated platelets was determined by flow cytometry and confocal laser scanning microscopy (CLSM). $2 \mathrm{~mL}$ platelets $\left(1.0 \times 10^{8} \mathrm{~mL}^{-1}\right)$ were seeded in 6-well plates and activated by incubation with $100 \mu \mathrm{L}$ of thrombin $(0.01 \mu \mathrm{M})$ for $10 \mathrm{~min}$. Unactivated platelets or thrombin-activated platelets were incubated with various FITC-labelled tPA-loaded liposomal formulations (equivalent tPA concentration of $0.2 \mathrm{mg} \mathrm{mL}^{-1}$ ). Free liposomes, which were not attached to platelets, were removed by centrifugation. Data for 1.0 $\times 10^{4}$ gated events were collected, and the analysis was carried out by a BD Fortessa II flow cytometer.

For CLSM, $2 \mathrm{~mL}$ platelets $\left(1.0 \times 10^{8} \mathrm{~mL}^{-1}\right)$ were seeded in 6-well plates, with a collagen-coated glass coverslip on the bottom of each well. After $30 \mathrm{~min}, 100 \mu \mathrm{L}$ of thrombin $(0.01 \mu \mathrm{M})$ was added onto the platelet-adhered coverslips to ensure activation of platelets. Unactivated platelets or thrombin-activated platelets were incubated with various FITC-labelled tPA-loaded liposomal formulations (equivalent tPA concentration of $0.2 \mathrm{mg}$ $\mathrm{mL}^{-1}$ ). The platelets were then fixed with $4.0 \%$ formaldehyde for $30 \mathrm{~min}$, followed by rinsing with pH 7.4 PBS buffer for three times. Subsequently, the resulting slides were mounted and observed with a Leica SP5 MP confocal microscope.

\subsection{In vitro drug release}

First, passive drug release was examined in $\mathrm{pH} 7.4 \mathrm{PBS}$ buffer at $37{ }^{\circ} \mathrm{C}$. The tPA-loaded liposomes (equivalent tPA dose of $0.5 \mathrm{mg} \mathrm{mL}^{-1}$ ) were placed into closed glass vials under shaking in a water bath at $37{ }^{\circ} \mathrm{C}$, and then ultracentrifuged for $1 \mathrm{~h}$ at each predetermined time 
interval. The released tPA was determined by measuring the tPA activity in the supernatant as described above.

Drug release was evaluated in the presence of activated platelets to confirm the tPA release specifically at the target site. $200 \mu \mathrm{L}$ of platelets $\left(1.0 \times 10^{8} \mathrm{~mL}^{-1}\right)$ were placed into a collagen-coated 96-well microplate and activated by treatment with $20 \mu \mathrm{L}$ thrombin $(0.01 \mu \mathrm{M})$. Unactivated platelets or thrombin-activated platelets were incubated with various tPA-loaded liposomal formulations (equivalent tPA dose of $0.5 \mathrm{mg} \mathrm{mL}^{-1}$ ). The released tPA in each well was determined by measuring the absorbance at $405 \mathrm{~nm}$ with a GloMax-Multi Microplate Multimode Reader (Promega, USA), as described above in the tPA activity assay. The percentage of tPA release was calculated by the following equation:

$$
\text { tPA release }(\%)=\frac{A-A_{C}}{A_{p}-A_{c}} \times 100
$$

where $A$ is the absorbance of platelet samples after incubation with the tPA-loaded liposomes; $A_{c}$ is the absorbance of negative control after incubation with the $\mathrm{pH}$ 7.4 PBS buffer only; $A_{p}$ is the absorbance of positive control after incubation with the tPA-loaded liposomes lysed by Triton X-100.

\subsection{Mechanisms of controlled drug release}

Study of activated-platelet-induced liposomal membrane destabilization: Destabilization of the membrane of the PEGylated, cRGD-coated liposome (PEG-cRGD-lip) was examined using a calcein dequenching assay [42-44]. Briefly, $200 \mu \mathrm{L}$ of resting or activated platelets $(1.0$ $\times 10^{8} \mathrm{~mL}^{-1}$ ) were added to $1.8 \mathrm{~mL}$ of the liposomal suspension encapsulated with $50 \mathrm{mM}$ calcein in pH 7.4 PBS buffer. The increase in calcein fluorescence due to calcein release from 
the destabilized PEG-cRGD-lip was detected with a Horiba FluoroMax-4 spectrofluorometer (HORIBA, USA) at $\lambda_{\mathrm{ex}}=488 \mathrm{~nm}$ and $\lambda_{\mathrm{em}}=535 \mathrm{~nm}$. The percentage of calcein fluorescence intensity was calculated relative to the maximal fluorescence intensity, which was recorded upon complete lysis of the calcein-loaded PEG-cRGD-lip by addition of Triton X-100 in the absence of platelets. Furthermore, in an inhibition experiment, $100 \mu \mathrm{g} \mathrm{mL} \mathrm{L}^{-1}$ eptifibatide, an $\alpha_{\mathrm{IIb}} \beta_{3}$ integrin inhibitor, was added to the suspension of activated platelets $5 \mathrm{~min}$ prior to mixing with the calcein-loaded PEG-cRGD-lip.

Confirmation of membrane fusion between the multifunctional liposome and activated platelets: A fluorescence resonance energy transfer (FRET) assay was employed to examine if membrane fusion was involved in the liposomal membrane destabilization upon interactions with resting or activated platelets $[45,46]$. Briefly, the membrane of PEG-cRGD-lip was incorporated with the donor NBD-PE ( $1 \mathrm{~mol} \%)$ and the acceptor Rhod-PE ( $1 \mathrm{~mol} \%) .200 \mu \mathrm{L}$ of resting or activated platelets $\left(1.0 \times 10^{8} \mathrm{~mL}^{-1}\right)$ were then added to $1.8 \mathrm{~mL}$ of the liposomal suspension in $\mathrm{pH}$ 7.4 PBS buffer. The donor NBD fluorescence was detected at $\lambda_{\mathrm{ex}}=465 \mathrm{~nm}$ and $\lambda_{\mathrm{em}}=520 \mathrm{~nm}$ with a Horiba FluoroMax-4 spectrofluorometer (HORIBA, USA). Furthermore, in an inhibition experiment, $100 \mu \mathrm{g} \mathrm{mL}^{-1}$ eptifibatide was added to the suspension of activated platelets to inhibit $\alpha_{\mathrm{II}} \beta_{3}$ integrins 5 min prior to mixing with PEG-cRGD-lip.

Measurement of tPA release in the presence of activated platelets at different concentrations: $200 \mu \mathrm{L}$ of platelets at a specific concentration were placed into each well of a collagen-coated 96-well microplate and activated by treatment with $20 \mu \mathrm{L}$ thrombin $(0.01 \mu \mathrm{M})$. Thrombin-activated platelets were incubated with the tPA-loaded, PEGylated, cRGD-coated 
liposome (tPA-PEG-cRGD-lip), and the percentage of released tPA in each well was determined according to the method described above.

\subsection{Determination of fibrin clot lysis}

Fibrin clot lysis by the tPA-loaded liposomes was measured by an agar plate assay [13, 41]. Briefly, $300 \mathrm{mg}$ of agar was dissolved into buffer mixture $(15 \mathrm{~mL}$ of $0.05 \mathrm{M}$ Tris- $\mathrm{HCl}$ buffer at $\mathrm{pH} 7.2$ and $5 \mathrm{~mL}$ of $0.025 \mathrm{M} \mathrm{CaCl}_{2}$ solution). Fibrinogen was dissolved into $10 \mathrm{~mL}$ of Tris- $\mathrm{HCl}$ buffer (0.05 M, pH 7.2). The agar solution was mixed with the fibrinogen solution, and then 10 $\mu \mathrm{L}$ of thrombin $(4.0 \mu \mathrm{M})$ was added under stirring for $1 \mathrm{~min}$. The resulting mixture was spread carefully on a transparent plastic plate and homogeneous gels were obtained at $37{ }^{\circ} \mathrm{C}$ after $3 \mathrm{~h}$. Four sample wells ( $0.8 \mathrm{~cm}$ diameter) were created in each plate and $10 \mu \mathrm{L}$ of plasminogen solution $\left(0.5 \mathrm{mg} \mathrm{mL}^{-1}\right)$ was then added into each sample well. PBS buffer, three plain liposomes (Lip, PEG-lip and PEG-cRGD-lip), as well as free tPA and the tPA-loaded liposomes (equivalent tPA dose of $0.5 \mathrm{mg} \mathrm{mL}^{-1}$ ) were added into respective sample wells and incubated at $37^{\circ} \mathrm{C}$. The area of the lysed zone in each well was measured to evaluate fibrin clot lysis.

\subsection{In vitro blood clot lysis}

Whole sheep blood together with $1.0 \mu \mathrm{M}$ thrombin and $5 \mathrm{mM} \mathrm{CaCl}_{2}(1: 0.1: 0.1, \mathrm{v}: \mathrm{v}: \mathrm{v})$ were placed into a pre-weighted glass tube under shaking at $37{ }^{\circ} \mathrm{C}$ for $3 \mathrm{~h}$ and then allowed to form a stable clot at $4{ }^{\circ} \mathrm{C}$ overnight. The initial weight of the blood clot was $0.793 \pm 0.068 \mathrm{~g}$, and its initial volume was calculated to be $156.84 \pm 3.46 \mathrm{~mm}^{3}$ by the following equation [47]:

$$
V\left(\mathrm{~mm}^{3}\right)=\frac{1}{2} \times \text { length }(\mathrm{mm}) \times \text { width }(\mathrm{mm}) \times \text { thickness }(\mathrm{mm})
$$


The various tPA-loaded liposomal formulations (at an equivalent tPA concentration of $0.5 \mathrm{mg}$ $\mathrm{mL}^{-1}$ ) were then added into the tubes. The clots treated with the $\mathrm{pH} 7.4$ PBS buffer alone or with the liposomes without tPA loading were selected as negative controls. The clots treated with free tPA at the same equivalent concentration were used as a positive control. At different time intervals after incubation at $37{ }^{\circ} \mathrm{C}$, the fluid in tubes was completely pipetted out and clots were then weighed again. The percentage of clot lysis was calculated by the following equation:

$$
\text { Clot lysis }(\%)=\frac{m}{m_{0}} \times 100
$$

where $m$ is the remaining blood clot weight after lysis; $m_{0}$ is the initial blood clot weight.

The time required for dissolving $50 \mathrm{wt} \%$ of the initial clot $\left(t_{50}\right)$ was also determined. At $1 \mathrm{~h}$ and $2 \mathrm{~h}$ post-treatment, the representative clot samples were photographed.

\subsection{Statistical Analysis}

All experiments were carried out in triplicate and the results were presented as mean values \pm standard deviation. The data were analyzed using two-tailed Student's $t$-test to assess the significant difference between groups. Differences were considered to be statistically significant when $p$ value was less than 0.05 .

\section{Results and discussion}

\subsection{Development and characterization of tPA-loaded, cRGD-coated, PEGylated}

\section{liposomes}

The tPA-loaded liposome surface modified with PEG and cRGD peptides, tPA-PEG-cRGD-lip, was fabricated according to the method depicted in Figure S2. The 
tPA-loaded liposome without surface modification, tPA-lip, and the tPA-loaded liposome surface modified by PEG only, tPA-PEG-lip, were prepared for comparison (Figure 1A). The molar percentage of cholesterol at $40 \mathrm{~mol} \%$ was chosen to prepare liposome formulations with desirable stability and minimal drug leakage according to our previous work [48]. Liposomal formulations were characterized for their particle size, morphology, PDI, zeta potential and encapsulation efficiency. As shown in Figure 1B, the DLS size of tPA-lip $(153.1 \pm 4.7 \mathrm{~nm})$ was similar to that of tPA-PEG-lip $(157.4 \pm 6.5 \mathrm{~nm})$ and tPA-PEG-cRGD-lip $(164.6 \pm 5.3 \mathrm{~nm})$. This suggests that neither PEGylation nor cRGD conjugation onto the liposomal surface caused any significant changes in particle size. The TEM image confirmed that tPA-PEG-cRGD-lip was spherical in shape (Figure 1C) and that the TEM particle size was consistent with the DLS result. As shown in Figure 1D, PDI values of the liposomal systems were in the range of 0.085 to 0.107 , suggesting that the formulations were monodisperse. Zeta potential values of the tPA-loaded liposomes were in the range of $-33.39 \pm 0.72$ to $-39.93 \pm$ $0.84 \mathrm{mV}$. The PEGylated systems displayed lower zeta potential probably due to the carbamate linker between DSPE and PEG, which was reported to introduce a negative charge to the liposomal surface at physiological $\mathrm{pH}$ [49]. The entrapment efficiency of fresh tPA-PEG-cRGD-lip was $33.4 \pm 3.6 \%$, higher than that for tPA-PEG-lip $(29.1 \pm 2.4 \%)$ and tPA-lip $(27.5 \pm 1.7 \%)$. The higher tPA entrapment in tPA-PEG-lip and tPA-PEG-cRGD-lip than in tPA-lip might be due to the increased hydrophilicity of the PEGylated lipids and the cRGD-modified lipids [50]. Stability of the tPA-loaded liposomes was examined by monitoring the size change and tPA leakage during storage at $4^{\circ} \mathrm{C}$. As shown in Figures $\mathbf{S 4}$ and S5, there was no significant liposomal size change or tPA leakage for all the formulations 
after 7 days of storage at $4{ }^{\circ} \mathrm{C}$. Upon extended storage up to 90 days, tPA-lip formed aggregation and showed a significantly increased hydrodynamic size, whilst no significant liposomal size change was observed for tPA-PEG-lip and tPA-PEG-cRGD-lip. It is also interesting to note that the tPA leakage from tPA-lip was up to $68.7 \pm 5.2 \%$ after 90 days of storage (Figure S5), whereas that for tPA-PEG-lip and tPA-PEG-cRGD-lip was only $15.1 \pm$ $3.7 \%$ and $12.5 \pm 3.7 \%$, respectively. The significantly enhanced stability of tPA-PEG-lip and tPA-PEG-cRGD-lip might be attributed to the increased repulsion between the liposomes with more negative surface charge and the shielding effect of the PEG coating, as compared with tPA-lip [50].
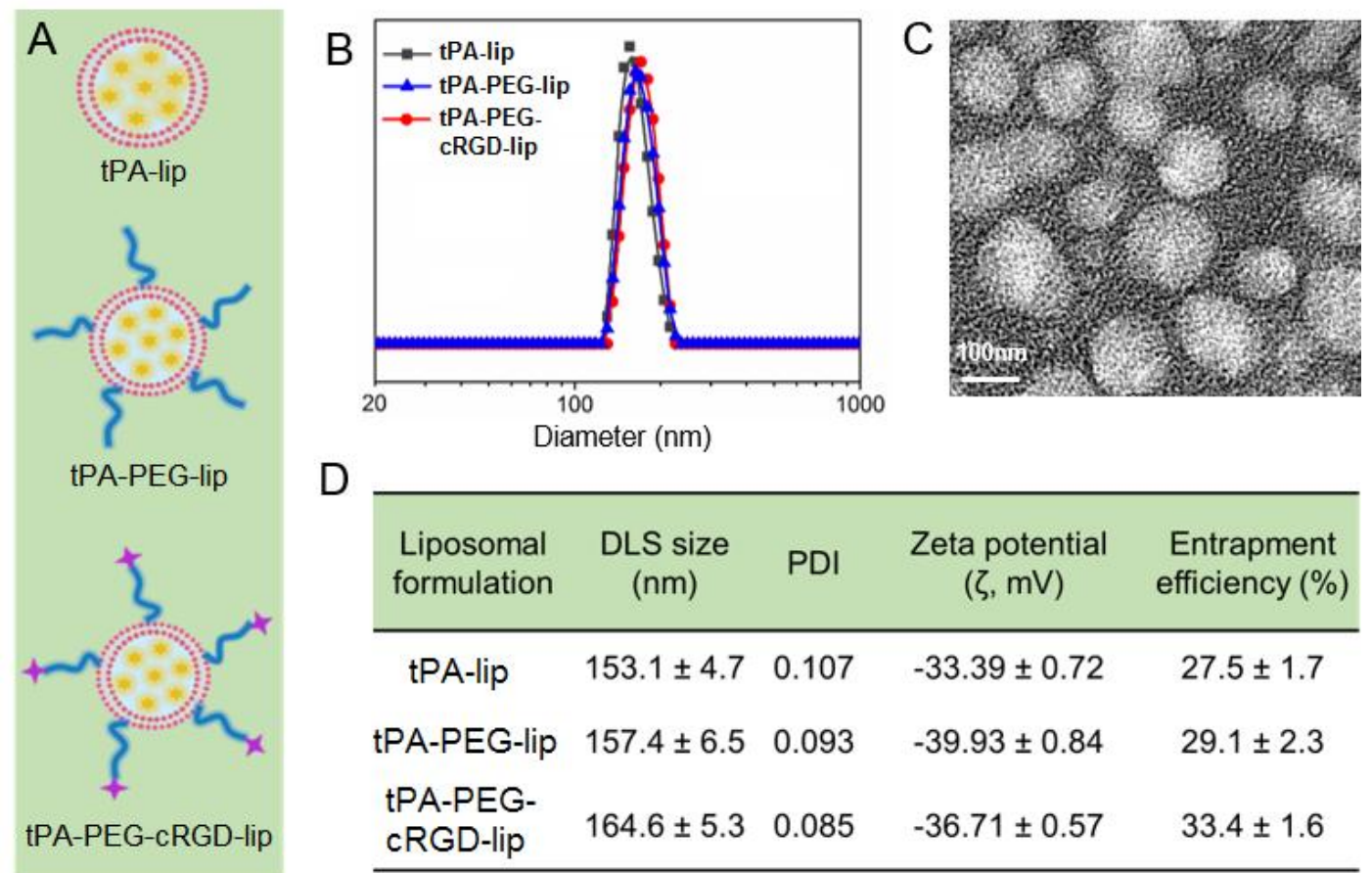

\begin{tabular}{lcccc}
\hline $\begin{array}{c}\text { Liposomal } \\
\text { formulation }\end{array}$ & $\begin{array}{c}\text { DLS size } \\
(\mathrm{nm})\end{array}$ & PDI & $\begin{array}{c}\text { Zeta potential } \\
(\zeta, \mathrm{mV})\end{array}$ & $\begin{array}{c}\text { Entrapment } \\
\text { efficiency }(\%)\end{array}$ \\
\hline tPA-lip & $153.1 \pm 4.7$ & 0.107 & $-33.39 \pm 0.72$ & $27.5 \pm 1.7$ \\
$\begin{array}{l}\text { tPA-PEG-lip } \\
\text { tPA-PEG- }\end{array}$ & $157.4 \pm 6.5$ & 0.093 & $-39.93 \pm 0.84$ & $29.1 \pm 2.3$ \\
cRGD-lip & $164.6 \pm 5.3$ & 0.085 & $-36.71 \pm 0.57$ & $33.4 \pm 1.6$ \\
\hline
\end{tabular}

Figure 1. (A) Illustration of three tPA-loaded liposomal formulations, tPA-lip, tPA-PEG-lip and tPA-PEG-cRGD-lip; (B) Typical intensity-weighted DLS plots of tPA-lip, tPA-PEG-lip and tPA-PEG-cRGD-lip in pH 7.4 PBS buffer; (C) TEM image of tPA-PEG-cRGD-lip; (D) Mean hydrodynamic sizes, PDIs, zeta potentials and encapsulation efficiencies of tPA-lip, tPA-PEG-lip and tPA-PEG-cRGD-lip. Data are presented as the average \pm standard deviation $(n=3)$. 


\subsection{Selective targeting to activated platelets}

A critical component of tPA-PEG-cRGD-lip is the targetable cRGD peptide, which can specifically bind to the $\alpha_{\mathrm{II}} \beta_{3}$ integrin expressed on activated platelets of thrombus and thus allow a selective accumulation of tPA at the clot site. Figure 2 compares the binding affinities of three FITC-labelled tPA-loaded liposomal formulations to resting platelets and activated platelets, respectively, by flow cytometry. As shown in Figures $\mathbf{2 A}$ and $\mathbf{2 B}$, the resting platelets treated with the FITC-labelled tPA-lip, tPA-PEG-lip and tPA-PEG-cRGD-lip displayed a similar level of fluorescence intensity, which was only slightly higher than that of the control resting platelets treated with pH 7.4 PBS buffer alone. This suggests that all the three liposomal formulations had a very low level of attachment to resting platelets. Figures 2C and 2D show that, even when the platelets were activated by thrombin, there was a negligible change in fluorescence intensity for the platelets treated with the FITC-labelled tPA-lip and tPA-PEG-lip. However, the FITC-labelled tPA-PEG-cRGD-lip bound more avidly to activated platelets, with about a 10-fold increase in fluorescence intensity as compared to the binding to resting platelets. These results suggest that cRGD peptides can efficiently facilitate the specific binding of the tPA-loaded liposomes to activated platelets [51]. The effect of cRGD coating density on the binding affinity of tPA-PEG-cRGD-lip to activated platelets was further investigated. As shown in Figures $2 \mathbf{E}$ and $\mathbf{2 F}$, the binding affinity of the FITC-labelled tPA-PEG-cRGD-lip to activated platelets gradually increased with increasing cRGD coating density from 0.3 to $0.7 \mathrm{~mol} \%$. This was followed by a considerable enhancement upon the increase of cRGD density to $1 \mathrm{~mol} \%$. The binding affinity reached a plateau at a cRGD density 
of $1 \mathrm{~mol} \%$ and a further introduction of cRGD on the liposomal surface would not enhance the targeting ability any further. This is consistent with a report that an intermediate ligand density provided optimal binding properties [52]. Thus, tPA-PEG-cRGD-lip with a cRGD density of 1 mol\% was chosen for the further studies herein.
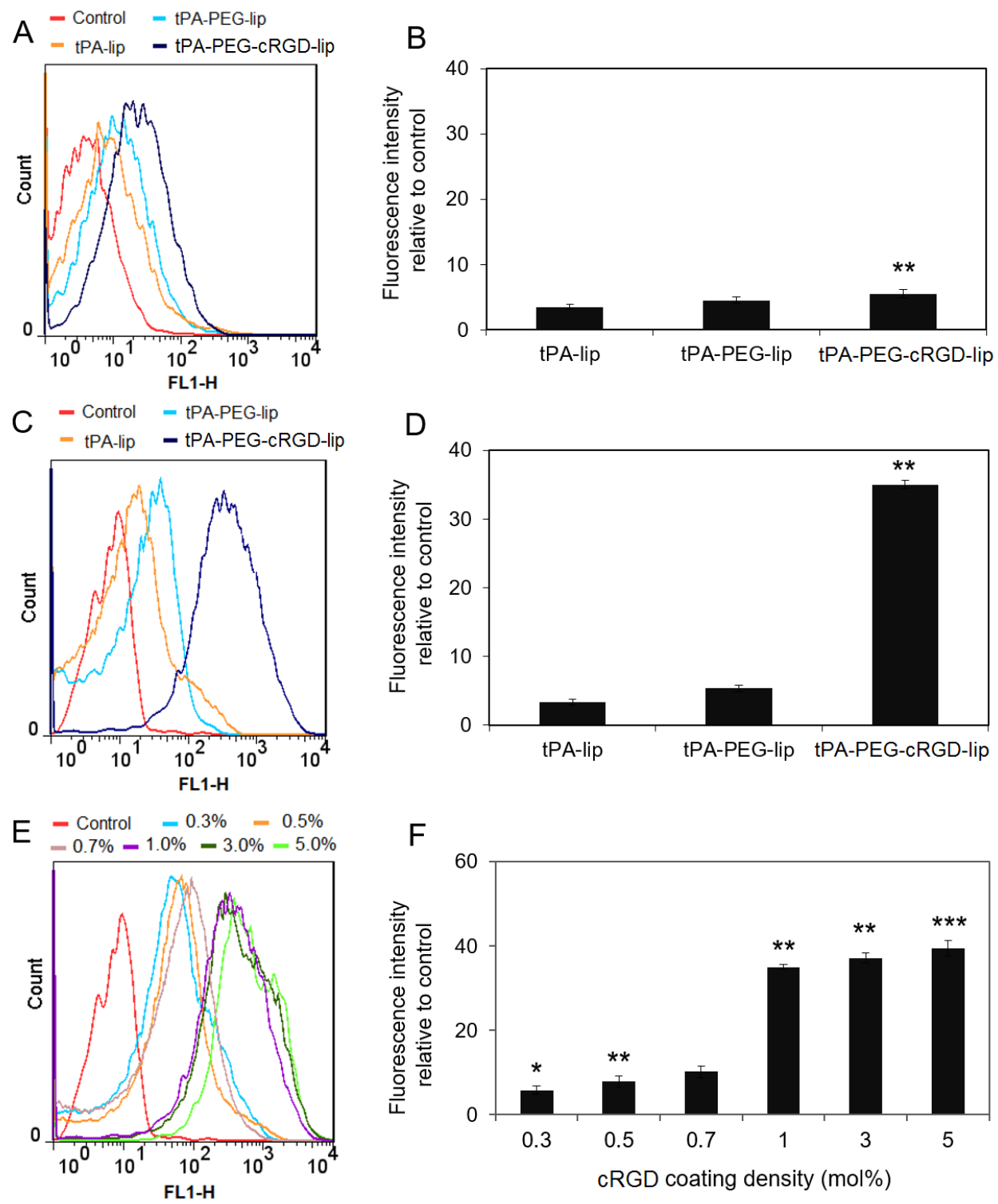

Figure 2. Flow cytometry histogram profiles of (A) resting platelets and (C) activated platelets incubated with the FITC-labelled tPA-lip, tPA-PEG-lip and tPA-PEG-cRGD-lip, respectively. The platelets incubated with pH 7.4 PBS buffer only were used as the negative control. Relative fluorescence intensities of (B) resting platelets and (D) activated platelets treated with 
the FITC-labelled tPA-lip, tPA-PEG-lip and tPA-PEG-cRGD-lip, respectively, relative to the control group. (E) Histogram profiles of activated platelets incubated with the FITC-labelled tPA-PEG-cRGD-lip containing the cRGD-DSPE densities of 0.3, 0.5, 0.7, 1.0, 3.0 and 5.0 mol\%, respectively; (F) Relative fluorescence intensities of activated platelets treated with the FITC-labelled tPA-PEG-cRGD-lip containing different cRGD peptide densities relative to the control group. All the FITC-tPA loaded liposomal solutions were used at the equivalent tPA concentration of $0.2 \mathrm{mg} \mathrm{mL}^{-1}$. Data are presented as the average \pm standard deviation $(\mathrm{n}=3)$. The single asterisk symbol $(*)$, double asterisk symbol $(* *)$ and triple asterisk symbol (***) denote to $p<0.05, p<0.01$ and $p<0.001$, respectively.

Furthermore, the selective binding ability of tPA-PEG-cRGD-lip was directly observed by CLSM. Both resting and activated platelets were observed in the bright field CLSM images shown in Figure S6. Figures 3A and 3B reveal that resting platelets treated with the FITC-labelled tPA-lip, tPA-PEG-lip and tPA-PEG-cRGD-lip only showed very weak green fluorescence. Interestingly, the CLSM images in Figure 3A show that tPA-PEG-cRGD-lip displayed significantly enhanced staining of activated platelets as compared to the FITC-labelled tPA-lip, tPA-PEG-lip without surface coating of cRGD peptides. Analysis of the mean fluorescence intensity (MFI) in those fluorescence images suggests that the enhancement in binding affinity of the FITC-labelled tPA-PEG-cRGD-lip to activated platelets was approximately 10-fold (Figure 3C). This is in good agreement with the flow cytometry results shown in Figure 2, further consolidating the ability of tPA-PEG-cRGD-lip to efficiently facilitate targeted drug delivery to activated platelets at a thrombus site. 

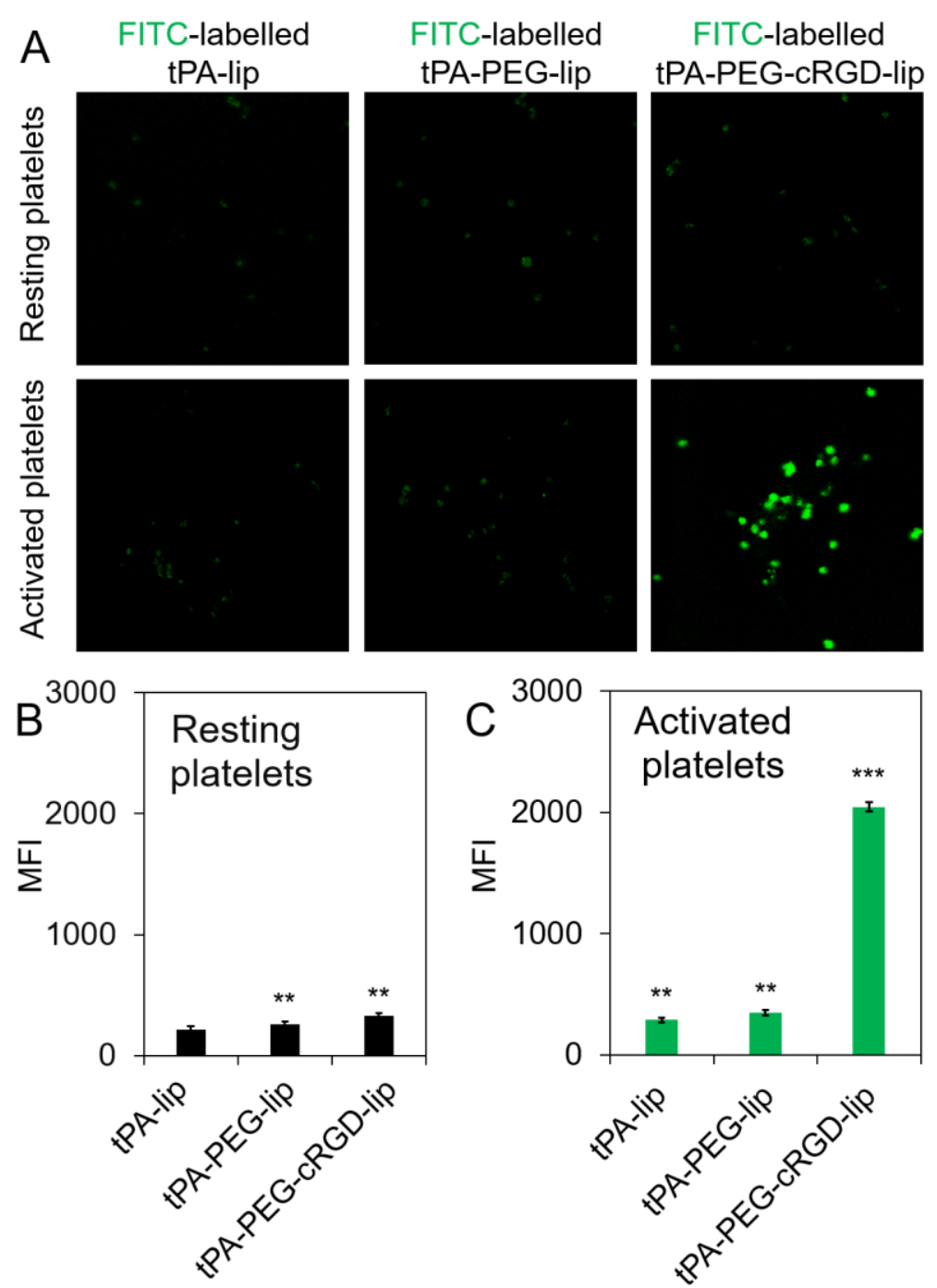

Figure 3. (A) CLSM images of resting platelets and activated platelets incubated with the FITC-labelled tPA-lip, tPA-PEG-lip and tPA-PEG-cRGD-lip, respectively; mean fluorescence intensity (MFI) in the fluorescence images of (B) resting platelets and (C) activated platelets incubated with the FITC-labelled tPA-lip, tPA-PEG-lip and tPA-PEG-cRGD-lip, respectively. All the FITC-tPA loaded liposomal solutions were used at the equivalent tPA concentration of $0.2 \mathrm{mg} \mathrm{mL}^{-1}$. Data are presented as the average \pm standard deviation $(\mathrm{n}=3)$. The double asterisk symbol $(* *)$ and triple asterisk symbol $(* * *)$ denote to $p<0.01$ and $p<0.001$, respectively.

\section{3. tPA release at the thrombus site}


The tPA release profiles of the liposomal formulations were further investigated to demonstrate the ability of tPA-PEG-cRGD-lip to release its drug payload specifically at the target site in the presence of activated platelets. First, passive tPA release from the liposomal formulations in $\mathrm{pH}$ 7.4 PBS buffer at $37{ }^{\circ} \mathrm{C}$ was examined. As shown in Figure S7, percentages of tPA released from tPA-lip, tPA-PEG-lip and tPA-PEG-cRGD-lip were all around 10\% after $6 \mathrm{~h}$. This indicates that the liposomal formulations containing $40 \mathrm{~mol} \%$ cholesterol could effectively retain tPA with relatively low passive drug release [48]. This is necessary for the entrapped tPA to survive in the blood circulation and then accumulate to activated platelets of a thrombus.
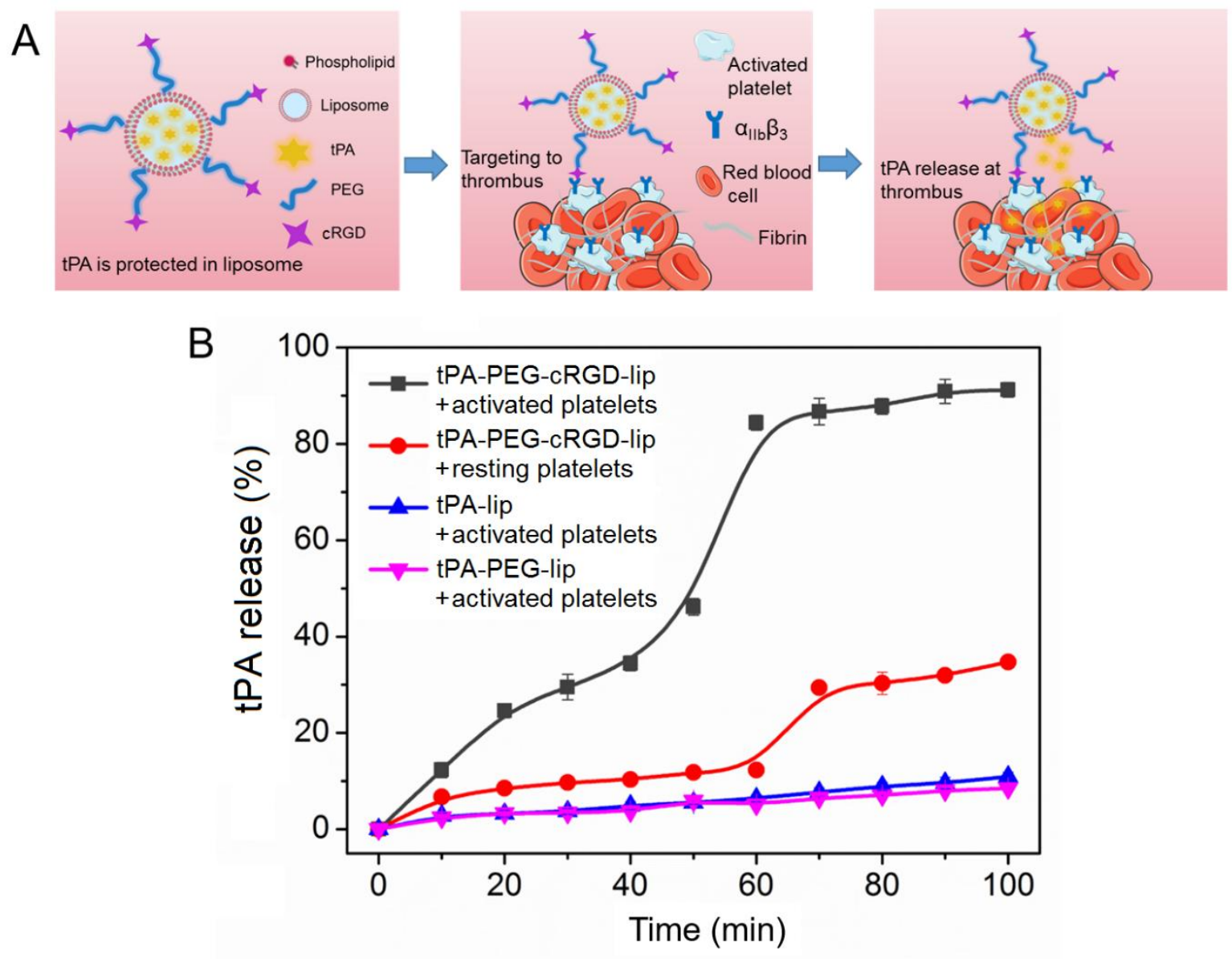

Figure 4. (A) Illustration of targeted delivery and release of tPA specifically at the thrombus site using tPA-PEG-cRGD-lip; (B) tPA release profiles of tPA-lip, tPA-PEG-lip and 
tPA-PEG-cRGD-lip after interaction with resting or activated platelets. All the tPA-loaded liposomal solutions were used at the equivalent tPA concentration of $0.5 \mathrm{mg} \mathrm{mL}^{-1}$. Data are presented as the average \pm standard deviation $(n=3)$.

Figure 4A illustrates the process of targeted delivery and controlled release of tPA specifically to the thrombus site. As shown in Figure $\mathbf{4 B}$, after binding of tPA-PEG-RGD-lip to activated platelets, over $90 \%$ of the entrapped tPA was released within $1 \mathrm{~h}$, which was considerably higher than the tPA release from tPA-lip and tPA-PEG-lip at less than 10\%. By comparison, when incubated with resting platelets, tPA-PEG-cRGD-lip released around $10 \%$ of the entrapped tPA in $1 \mathrm{~h}$ and this was then increased to a plateau of approximately $30 \%$ after 70 min, which might be due to its slightly higher binding affinity than tPA-lip and tPA-PEG-lip

(Figures 2B and 3B). These results indicate that drug release from the liposomal formulation was preferably induced by the interaction of cRGD peptides on the surface of tPA-PEG-cRGD-lip with the $\alpha_{\mathrm{IIb}} \beta_{3}$ on the surface of activated platelets.

\subsection{Mechanisms of controlled release of tPA}

The efficient tPA release mediated by the specific interactions between tPA-PEG-cRGD-lip and activated platelets prompted us to examine the mechanisms controlling the release of tPA. It has been reported that liposomes containing targeting motifs had an ability to bind to the herpes simplex virus (HSV) or HSV-infected cells, which led to the release of the liposomal content as a result of liposomal membrane destabilization $[53,54]$. Inspired by this, a calcein dequenching assay [42-44] was used to examine if payload was released due to the liposomal membrane destabilization of PEG-cRGD-lip upon its interactions with activated platelets. As 
shown in Figure 5B, when calcein was encapsulated into PEG-cRGD-lip at a concentration of $50 \mathrm{mM}$, approximately $70 \%$ of its fluorescence intensity was self-quenched initially. After incubation of the calcein-loaded PEG-cRGD-lip with PBS buffer only (i.e. no platelets) or resting platelets, no significant change in the relative calcein fluorescence intensity was observed, which suggests that calcein was still entrapped within the liposome. However, after binding of the calcein-loaded PEG-cRGD-lip to activated platelets, the relative calcein fluorescence intensity was enhanced rapidly and considerably within $15 \mathrm{~min}$, indicative of the efficient calcein release as a result of liposomal membrane destabilization. By contrast, upon inhibition of $\alpha_{\mathrm{II}} \beta_{3}$ integrins by eptifibatide the relative calcein fluorescence intensity of the calcein-loaded PEG-cRGD-lip in the presence of activated platelets was remarkably reduced to the similar level with that of the calcein-loaded PEG-cRGD-lip in the presence of PBS buffer only or resting platelets. This suggests that the liposomal content release can result from the specific binding of cRGD peptides on the liposomal surface with $\alpha_{\mathrm{IIb}} \beta_{3}$ integrins on the activated platelet surface, which drives PEG-cRGD-lip to be sufficiently close to activated platelets, a prerequisite of the liposomal membrane destabilization.

In addition, a fluorescence resonance energy transfer (FRET) assay [45, 46] was used to investigate if fusion between the membrane of PEG-cRGD-lip and the membrane of activated platelets was involved in the liposomal membrane destabilization process. As shown in Figure $\mathbf{5 A}$, the membrane of PEG-cRGD-lip was incorporated with NBD-PE (1 mol\%) and Rhod-PE (1 mol\%). The efficiency of energy transfer between the donor fluorophore NBD and the acceptor fluorophore Rhod was dependent on their surface density and distance on the liposomal membrane. Membrane fusion between PEG-cRGD-lip and activated platelets would 
lead to a decrease in the fluorophore surface density and a subsequent increase in the fluorescence intensity of the donor fluorophore, which was confirmed by the remarkable enhancement in the NBD fluorescence intensity after 30 min of incubation (Figures S8 and 5C). As shown in Figure 5C, the NBD fluorescence intensity was further increased significantly with extending the incubation time. By comparison, when the PEG-cRGD-lip containing NBD-PE and Rhod-PE was incubated with PBS buffer only or resting platelets, no significant enhancement in the NBD fluorescence was observed, indicative of negligible membrane fusion. It is interesting to note that, once $\alpha_{\mathrm{IIb}} \beta_{3}$ integrins expressed on the surface of activated platelets were inhibited by eptifibatide, there was only a marginal increase in the NBD fluorescence intensity. This suggests that the selective interactions of cRGD peptides with $\alpha_{\mathrm{IIb}} \beta_{3}$ integrins can trigger the membrane fusion, leading to controlled release of the liposomal content.

Furthermore, triggered release of tPA from tPA-PEG-cRGD-lip was evaluated after incubation with activated platelets at different concentrations. As shown in Figure S9, the efficiency of tPA release was dependent on the concentration of activated platelets added. With increasing the activated platelet concentration, a significant increase in tPA release was observed after incubation for $15 \mathrm{~min}$. This indicates that the release of tPA can be readily tuned by the number of activated platelets. 


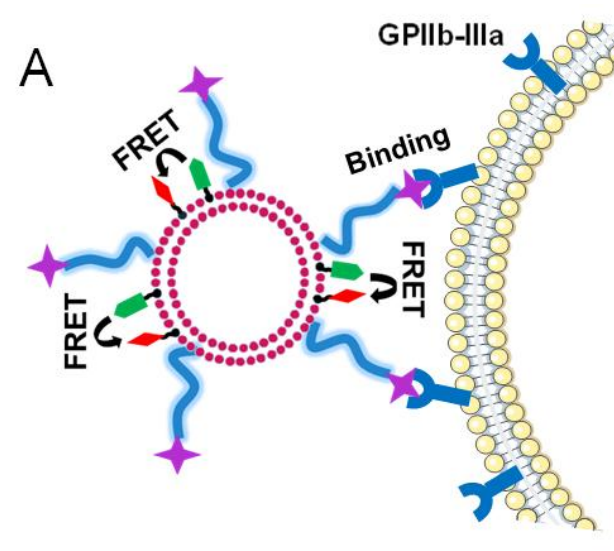

B

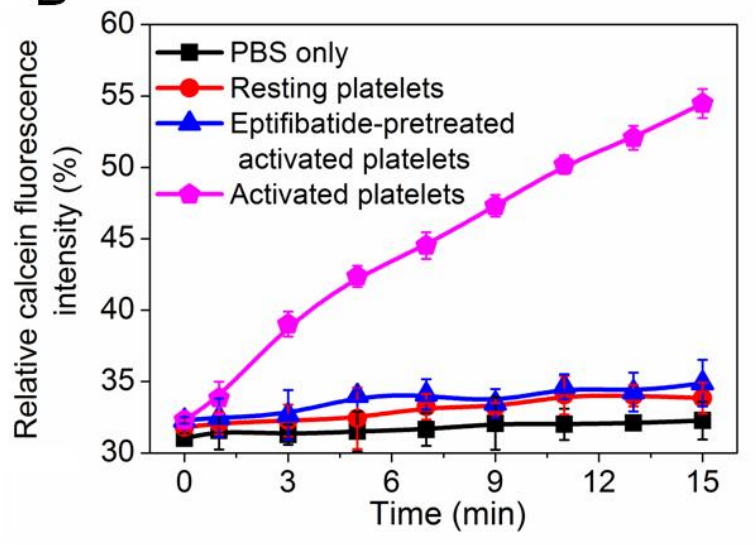

Activated platelet

membrane

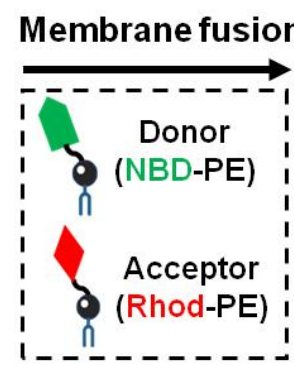

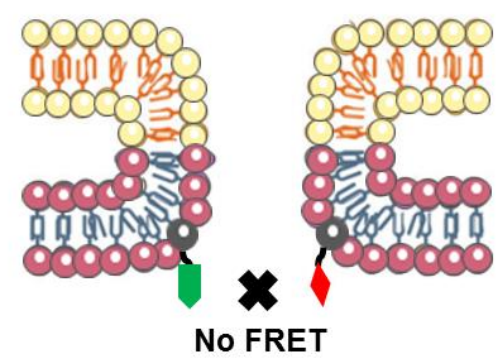

No FRET

C

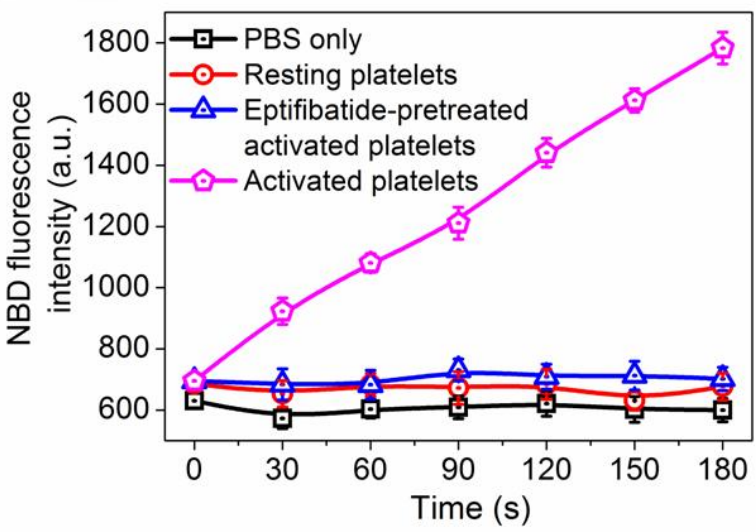

Figure 5. (A) Schematic illustration of the membrane fusion of PEG-cRGD-lip (containing 1 mol\% NBD-PE and 1 mol\% Rhod-PE) with activated platelets through selective interactions. (B) Relative calcein fluorescence intensity measured after incubation of the calcein-loaded PEG-cRGD-lip with PBS buffer only, resting platelets, activated platelets and eptifibatide-pretreated activated platelets, respectively, at various time intervals. (C) NBD fluorescence intensity after incubation of PEG-cRGD-lip (containing $1 \mathrm{~mol} \%$ NBD-PE and 1 mol\% Rhod-PE) with PBS buffer only, resting platelets, activated platelets and eptifibatide-pretreated activated platelets, respectively, at various time intervals. Data are presented as the average \pm standard deviation $(n=3)$.

\subsection{In vitro fibrin clot lysis}

tPA is a fibrinolytic enzyme responsible for breaking up blood clots via fibrin lysis. Effective release of tPA from tPA-PEG-cRGD-lip was confirmed through the interaction of homing peptides with activated platelets. This prompted the use of a fibrin clot model to investigate the 
fibrinolytic activity of tPA-PEG-cRGD-lip in the presence of activated platelets. In this model as shown in Figure 6A, the degree of fibrin lysis was monitored visually by measuring the diameter and area of the lysis ring around each sample well [55,56]. Firstly, three plain liposomes without tPA (i.e. Lip, PEG-lip and PEG-cRGD-lip), and PBS buffer alone were placed into respective sample wells and incubated at $37{ }^{\circ} \mathrm{C}$. As shown in Figure 6B, no significant change of the fibrin clots was observed even up to $24 \mathrm{~h}$ of incubation, suggesting that none of the components of the liposomal formulations and PBS buffer broke up the fibrin clots themselves, and that fibrin lysis was solely due to the fibrinolytic protein. Fibrinolytic activity of tPA-PEG-cRGD-lip was then evaluated in the presence of activated platelets to confirm the selective fibrin lysis at the target site. As shown in Figures $6 \mathbf{C} \sim \mathbf{6 E}$, tPA-PEG-cRGD-lip was placed into the respective sample wells containing the PBS buffer alone, resting platelets or thrombin-activated platelets and incubated for $0.5 \mathrm{~h}, 1 \mathrm{~h}, 1.5 \mathrm{~h}$ and 2 h, respectively. For the fibrin clot in the presence of PBS buffer (Figure 6C) and resting platelets (Figure 6D), only small lysis rings were observed until $2 \mathrm{~h}$ of incubation, which might be due to the marginal tPA release from tPA-PEG-cRGD-lip. Interestingly, upon treatment of the fibrin clot with tPA-PEG-cRGD-lip and activated platelets (Figure 6E), a clear clot lysis zone around the sample well was observed after $0.5 \mathrm{~h}$ and the diameter of the lysis ring increased over time, up to $2 \mathrm{~h}$. This could be ascribed to the effective release of a large amount of tPA from tPA-PEG-cRGD-lip, induced by its selective binding to activated platelets. The areas of fibrin clot lysis rings were calculated and plotted against time in Figure 6F. It was shown that, in the presence of activated platelets, tPA-PEG-cRGD-lip caused significant fibrin lysis within $2 \mathrm{~h}$; considerably higher than that in the sample wells containing 
tPA-PEG-cRGD-lip and PBS buffer or resting platelets. Figure 6G shows that the area of lysis ring caused by tPA-PEG-cRGD-lip $\left(0.532 \pm 0.072 \mathrm{~cm}^{2}\right)$ in the presence of activated platelets was smaller than that caused by free tPA $\left(1.368 \pm 0.101 \mathrm{~cm}^{2}\right)$ at initial $0.5 \mathrm{~h}$. This could be ascribed to the incomplete tPA release (approximately 30\%) from tPA-PEG-cRGD-lip at initial 0.5 h, as shown in Figure 4B. However, when tPA-PEG-cRGD-lip was added into the sample well containing activated platelets for $24 \mathrm{~h}$ of incubation at $37^{\circ} \mathrm{C}$ to ensure complete tPA release, the degree of fibrin lysis caused by tPA-PEG-cRGD-lip was $97.4 \pm 1.7 \%$ relative to that caused by free tPA (Figure 6H), suggesting that the tPA encapsulated inside the liposome retained its functionality.

A
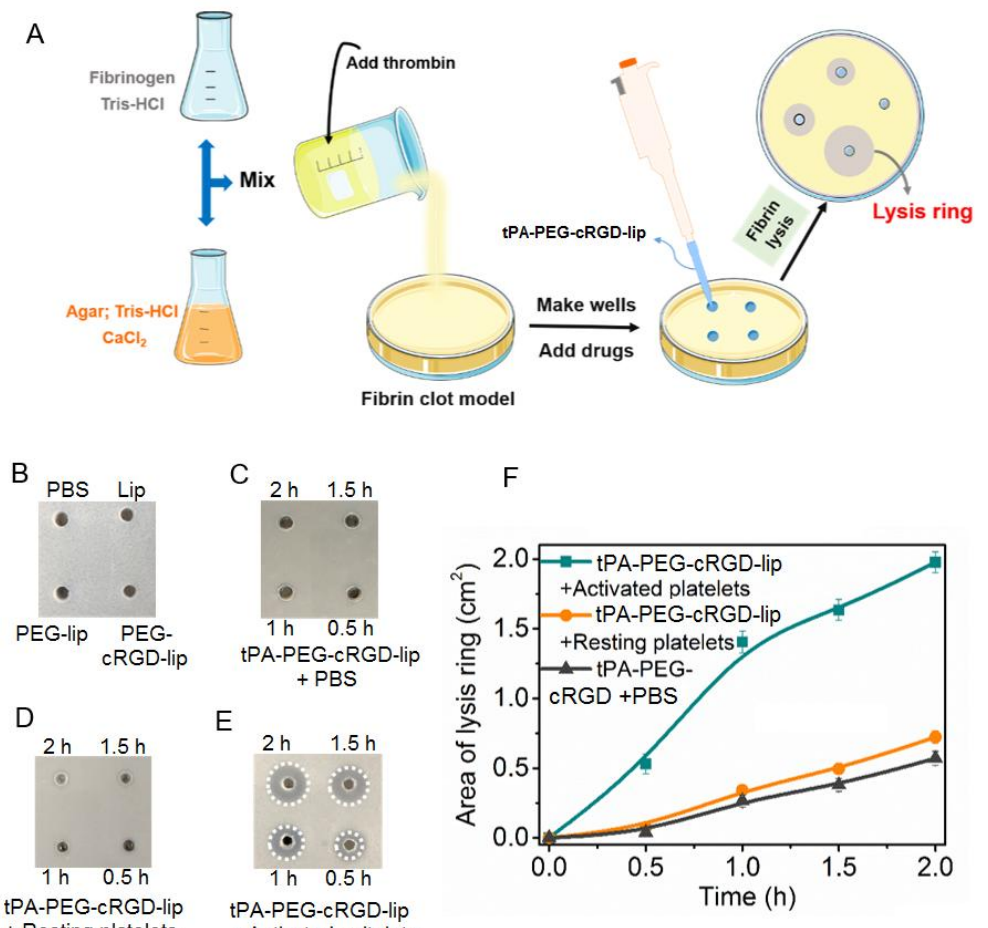

E
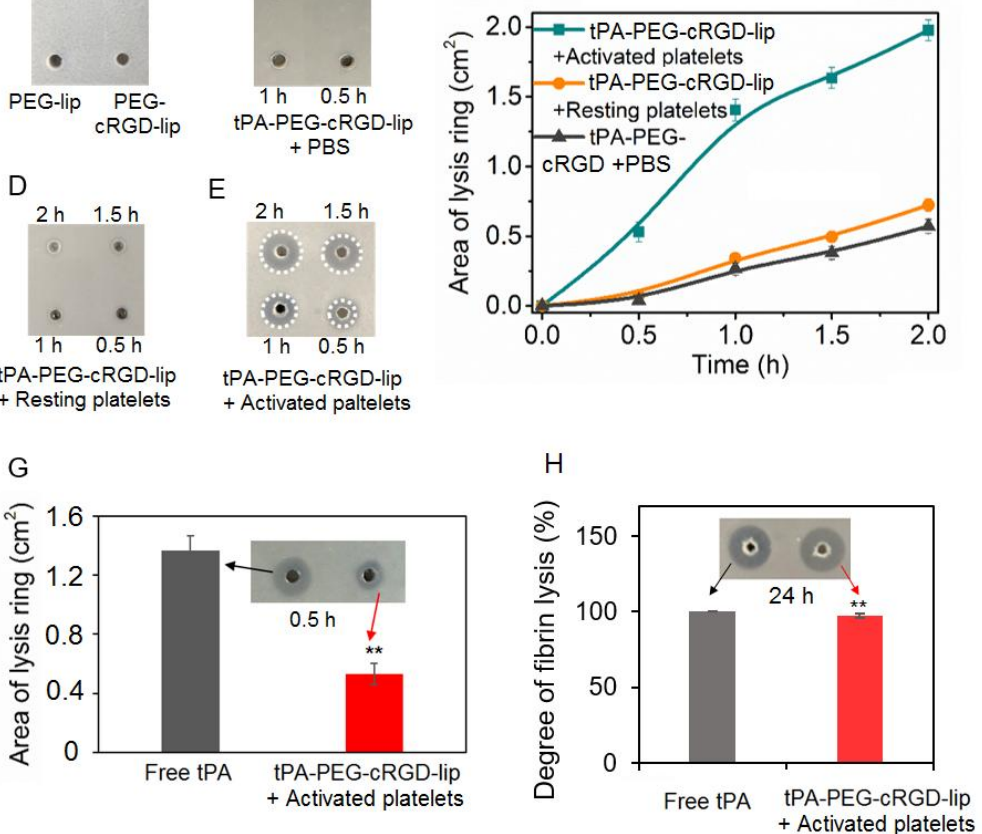
Figure 6. (A) Schematic illustration of the preparation of the fibrin clot model to investigate fibrinolytic activity; (B) Typical photograph of the fibrin clot treated with $\mathrm{pH} 7.4$ PBS buffer, Lip, PEG-lip and PEG-cRGD-lip solutions respectively at $37{ }^{\circ} \mathrm{C}$ for $24 \mathrm{~h}$; (C) Typical photograph of the fibrin clot treated with tPA-PEG-cRGD-lip in the presence of $\mathrm{pH} 7.4$ PBS buffer at $37{ }^{\circ} \mathrm{C}$ for $0.5,1,1.5$ and $2 \mathrm{~h}$, respectively; (D) Typical photograph of the fibrin clot treated with tPA-PEG-cRGD-lip in the presence of resting platelets at $37{ }^{\circ} \mathrm{C}$ for $0.5,1,1.5$ and

$2 \mathrm{~h}$, respectively; (E) Typical photograph of the fibrin clot treated with tPA-PEG-cRGD-lip in the presence of activated platelets at $37^{\circ} \mathrm{C}$ for $0.5 \mathrm{~h}, 1 \mathrm{~h}, 1.5 \mathrm{~h}$ and $2 \mathrm{~h}$, respectively; (F) Areas of fibrin clot lysis rings calculated from the photographs in $\mathrm{C}-\mathrm{E} ;(\mathrm{G})$ Areas of fibrin clot lysis rings calculated from the inset photograph of the fibrin clots treated with free tPA and tPA-PEG-cRGD-lip, respectively in the presence of activated platelets for $0.5 \mathrm{~h}$; $(\mathrm{H})$ The degree of fibrin lysis of free tPA and tPA-PEG-cRGD-lip in the presence of activated platelets, as measured from the inset photograph of the fibrin clot treated with free IPA and tPA-PEG-cRGD-lip respectively in the presence of activated platelets at $37{ }^{\circ} \mathrm{C}$ for $24 \mathrm{~h}$. The degree of fibrin lysis by tPA-PEG-cRGD-lip is a percentage of the area of lysis ring caused by tPA-PEG-cRGD-lip relative to that caused by free tPA (with the degree of fibrin lysis by free tPA normalized as 100\%). tPA-PEG-cRGD-lip and free tPA solutions had the equivalent tPA concentration of $0.5 \mathrm{mg} \mathrm{mL}^{-1}$. Data are presented as the average \pm standard deviation $(\mathrm{n}=3)$. The double asterisk symbol $(* *)$ denotes to $p<0.01$.

\subsection{In vitro blood clot lysis}

The high efficiency of tPA-PEG-cRGD-lip in targeted delivery and effective release of tPA to activated platelets and the subsequent fibrin clot lysis led us to evaluate its ability to selectively and efficiently dissolve blood clots. Blood clots were randomly divided into eight groups and incubated with three liposomes without tPA (i.e. Lip, PEG-lip and PEG-cRGD-lip), three tPA-loaded liposomes (i.e. tPA-lip, tPA-PEG-lip and tPA-PEG-cRGD-lip), PBS buffer alone, and free tPA solutions, respectively. The representative clot photographs at 1 and $2 \mathrm{~h}$ post-treatment are displayed in Figure 7A. It was clearly found that no clot was observed after 
incubation with tPA-PEG-cRGD-lip solution for $2 \mathrm{~h}$, similar to the clot sample treated with free tPA. By contrast, the remnant clots were visible to the naked eye after $2 \mathrm{~h}$ of treatment with tPA-lip, tPA-PEG-lip, three plain liposomes (Lip, PEG-lip and PEG-cRGD-lip in Figure S10), and PBS buffer. Clot weight monitoring was used to evaluate the efficacy of thrombolytic therapy. As shown in Figure 7B, the clot-lytic activity of tPA-PEG-cRGD-lip was lower than free tPA in the initial timepoints, which could be ascribed to the time required for tPA release from the multifunctional liposomes, as shown in Figure 4B. However, the difference between tPA-PEG-cRGD-lip and free tPA was not considerable (approximately 15\%). This is probably because the clot lysis induced by tPA is a gradual process, as reported by other researchers [57, 58]. However, after $75 \mathrm{~min}$ of treatment when majority of tPA was released from tPA-PEG-cRGD-lip (Figure 4B), the liposomal tPAs, like free tPA, caused almost the complete blood clot lysis. By comparison, the degree of clot dissolution by the tPA-loaded liposomal formulations without cRGD coating (i.e. tPA-lip and tPA-PEG-lip) was found to be no more than $50 \%$ after incubation for $2 \mathrm{~h}$. In addition, the clot lytic activity of three liposomes without tPA loading (i.e. Lip, PEG-lip and PEG-cRGD-lip) was found to be similar to that of PBS buffer (negative control), confirming that all components of the liposomal formulations did not induce clot lysis. Furthermore, $t_{50}$, the time required for dissolving $50 \%$ of the initial clot was recorded in Figure 7C. It was found that tPA-PEG-cRGD-lip dissolved $50 \%$ of the initial clot in 48.2 min, whilst $t_{50}$ for tPA-lip and tPA-PEG-lip was 122.3 and $128.1 \mathrm{~min}$, respectively. The much shorter $t_{50}$ recorded for tPA-PEG-cRGD-lip was likely due to much more efficient and quicker release of tPA at the thrombus site specifically. 


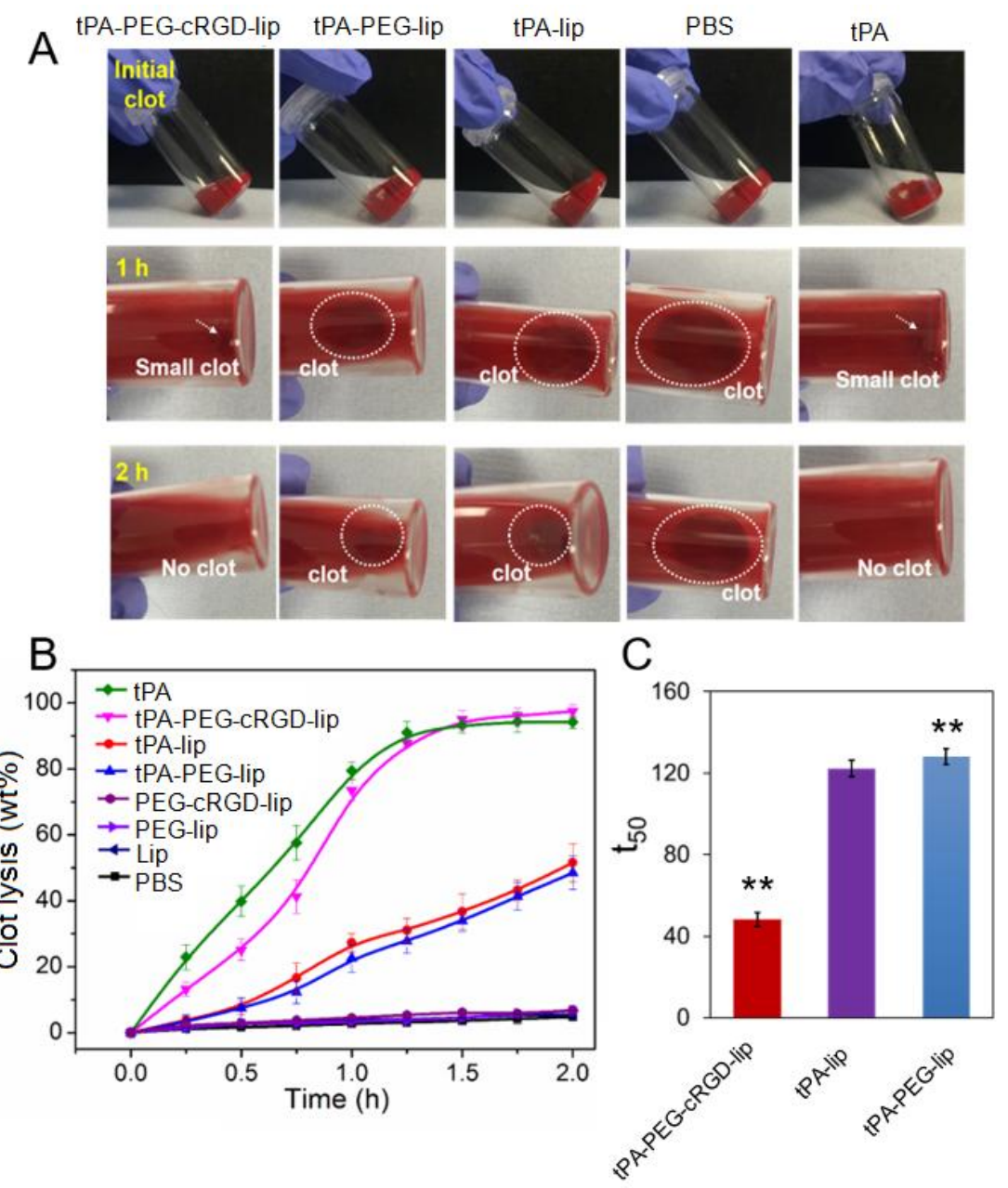

Figure 7. (A) Observations of clot dissolution after treatment with tPA-PEG-cRGD-lip, tPA-PEG-lip, tPA-lip, PBS buffer and free tPA for 1 and $2 \mathrm{~h}$, respectively; (B) Time-dependent in vitro clot lysis after treatment with tPA-PEG-cRGD-lip, tPA-PEG-lip, tPA-lip, liposomes without tPA (PEG-cRGD-lip, PEG-lip and Lip), PBS buffer and free tPA; (C) Comparison of $t_{50}$, the time required for dissolving $50 \%$ of the initial clot, of tPA-PEG-cRGD-lip, tPA-PEG-lip and tPA-lip. All the tPA-containing sample solutions were used at the equivalent tPA concentration of $0.5 \mathrm{mg} \mathrm{mL}^{-1}$. Data are presented as the average \pm standard deviation $(\mathrm{n}=$ 3). The double asterisk symbols $(* *)$ denote to $p<0.01$.

\section{Conclusions}

In summary, an activated-platelet-sensitive nanoliposome, tPA-PEG-cRGD-lip, was successfully constructed and characterized for selective thrombolysis through targeted delivery 
and controlled release of tPA to the blood clot. The tPA-loaded, cRGD-coated, PEGylated liposome had a small size ( $160 \mathrm{~nm}$ in diameter), narrow size distribution and good stability. Flow cytometry and CLSM measurements showed a highly specific binding of tPA-PEG-cRGD-lip to activated platelets. In addition, it was demonstrated that efficient tPA release from tPA-PEG-cRGD-lip was induced through liposomal membrane destabilization involving membrane fusion upon selective binding of the liposome to activated platelets. The release of tPA could be readily controlled by varying the concentration of activated platelets. The presence of activated platelets was demonstrated to cause significant fibrin clot lysis. Furthermore, tPA-PEG-cRGD-lip had considerably higher thrombolytic activity and significantly reduced the time required for clot lysis compared to non-cRGD-coated liposomes. Therefore, this nano-sized, activated-platelet-sensitive liposomal system could facilitate targeted thrombolytic therapy whilst minimising undesirable side effects. This new approach holds significant promise for application in the treatment of various cardiovascular diseases.

\section{Acknowledgements}

This research was funded by the National Institute for Health Research (NIHR) Biomedical Research Centre based at Imperial College Healthcare NHS Trust and Imperial College London. The views expressed are those of the authors and not necessarily those of the NHS, the NIHR or the Department of Health. A.D.H. receives support from the NIHR University College London Hospitals Biomedical Research Centre, and works in a unit that receives support from the UK Medical Research Council (Programme Code MC_UU_12019/1). The authors thank Dr Ecaterina Ware for the assistance in TEM measurement. The authors would 
also like to thank Dr Jessica Rowley for access to flow cytometry, Dr Andreas Bruckbauer for access to laser scanning confocal microscope, and Ms Patricia Carry for access to Analytical Services in the Department of Chemical Engineering at Imperial College London.

\section{Conflicts of Interest}

The authors declare no conflict of interest.

\section{Supplementary Data}

Supplementary data to this article can be found online at https://xxx

${ }^{1} \mathrm{H}$ NMR spectrum of DSPE-PEG-cRGD, illustration of the preparation process of tPA-PEG-cRGD-lip, illustration of differential centrifugation for platelet isolation, variations in the liposomal size and tPA leakage during storage at $4{ }^{\circ} \mathrm{C}$, bright field images of resting and activated platelets incubated with tPA-loaded liposomes, passive tPA release profiles of liposomal formulations in $\mathrm{pH}$ 7.4 PBS buffer at $37{ }^{\circ} \mathrm{C}$, fluorescence spectra of PEG-cRGD-lip (containing $1 \mathrm{~mol} \% \mathrm{NBD}-\mathrm{PE}$ and $1 \mathrm{~mol} \%$ Rhod-PE) before and after incubation with activated platelets, tPA release after incubation of tPA-PEG-cRGD-lip with different concentrations of activated platelets, and observation of blood clot dissolution after treatment with Lip, PEG-lip, and PEG-cRGD-lip.

Data underlying this Article can be accessed on Zenodo (www.zenodo.org) at https://doi.org/ 10.5281/zenodo.1290818 and is openly available under the Creative Commons CC-BY license. 


\section{REFERENCES}

[1] G. Lippi, M. Franchini, G. Targher, Arterial thrombus formation in cardiovascular disease, Nat. Rev. Cardiol. 8 (2011) 502-512.

[2] V. Fuster, L. Badimon, J. J. Badimon, J. H. Chesebro, The pathogenesis of coronary artery disease and the acute coronary syndromes, N. Engl. J. Med. 326 (1992) 310-318.

[3] V. Marder, Thrombolytic therapy, Blood Rev. 15 (2001) 143-157.

[4] G. Damaschun, H. Damaschun, K. Gast, D. Gerlach, R. Misselwitz, H. Welfle, D. Zirwer, Streptokinase is a flexible multi-domain protein, Eur. Biophys. J. 20 (1992) 355-361.

[5] D. B. Baruah, R. N. Dash, M. R. Chaudhari, S. S. Kadam, Plasminogen activators: a comparison, Vascul. Pharmacol. 44 (2006) 1-9.

[6] G. Cesarman-Maus, K. A. Hajjar, Molecular mechanisms of fibrinolysis, Br. J. Haematol. 129 (2005) 307-321.

[7] W. L. Chandler, M. C. Alessi, M. F. Aillaud, P. Henderson, P. Vague, I. Juhan-Vague, Clearance of tissue plasminogen activator (TPA) and TPA/plasminogen activator inhibitor Type 1 (PAI-1) complex: relationship to elevated TPA antigen in patients with high PAI-1 activity levels, Circulation 96 (1997) 761-768.

[8] O. Matsuo, An ideal thrombolytic and antithrombotic agent, J. Thromb. Haemost. 3 (2005) 2154-2155.

[9] J. K. Leach, E. Patterson, E. A. O’Rear, Distributed intraclot thrombolysis: mechanism of accelerated thrombolysis with encapsulated plasminogen activators, J. Thromb. Haemost. 2 (2004) 1548-1555. 
[10] C. R. Mayer, R. Bekeredjian, Ultrasonic gene and drug delivery to the cardiovascular system, Adv. Drug Deliv. Rev. 60 (2008) 1177-1192.

[11] V. Saxena, C. G. Johnson, A. H. Negussie, K. V. Sharma, M. R. Dreher, B. J. Wood, Temperature-sensitive liposome-mediated delivery of thrombolytic agents, Int. J. Hyperthermia 31 (2015) 67-73.

[12] J. H. Berger, S. V. Pizzo, Preparation of polyethylene glycol-tissue plasminogen activator adducts that retain functional activity: characteristics and behavior in three animal species. Blood 71 (1988) 1641-1647.

[13] J.Y. Kim, J. K. Kim, J. S. Park, Y. Byun, C. K. Kim, The use of PEGylated liposomes to prolong circulation lifetimes of tissue plasminogen activator, Biomaterials 30 (2009) $5751-5756$.

[14] J. Myerson, L. He, G. Lanza, D. Tollefsen, S. Wickline, Thrombin-inhibiting perfluorocarbon nanoparticles provide a novel strategy for the treatment and magnetic resonance imaging of acute thrombosis, J. Thromb. Haemostasis 9 (2011) 1292-1300.

[15] T. W. Chung, S. S. Wang, W. J. Tsai, Accelerating thrombolysis with chitosan-coated plasminogen activators encapsulated in poly-(lactide-co-glycolide) (PLGA) nanoparticles, Biomaterials 29 (2008) 228-237.

[16] G. Huang, G, Z. Zhou, R. Srinivasan, M. S. Penn, K. Kottke-Marchant, R. E. Marchant, A. S. Gupta, Affinity manipulation of surface-conjugated RGD peptide to modulate binding of liposomes to activated platelets, Biomaterials 29 (2008) 1676-1685.

[17] Z. Wu, H. Chen, D. Li, J. L. Brash, Tissue plasminogen activator-containing polyurethane surfaces for fibrinolytic activity, Acta Biomater. 7 (2011) 1993-1998. 
[18] S. Jin, Y. Wang, H. Zhu, Y. Wang, S. Zhao, M. Zhao, J. Liu, J. Wu, W. Gao, S. Peng, Nanosized aspirin-arg-gly-asp-val: delivery of aspirin to thrombus by the target carrier arg-gly-asp-val tetrapeptide, ACS Nano 7 (2013) 7664-7673.

[19] B. Vaidya, G. P. Agrawal, S. P. Vyas, Functionalized carriers for the improved delivery of plasminogen activators, Int. J. Pharm. 424 (2012) 1-11.

[20] N. Korin, M. Kanapathipillai, B. D. Matthews, M. Crescente, A. Brill, T. Mammoto, K. Ghosh, S. Jurek, S. A. Bencherif, D. Bhatta, Shear-activated nanotherapeutics for drug targeting to obstructed blood vessels, Science 337 (2012) 738-742.

[21] R. Cheng, W. Huang, L. Huang, B. Yang, L. Mao, K. Jin, Q. ZhuGe, Y. Zhao, Acceleration of tissue plasminogen activator-mediated thrombolysis by magnetically powered nanomotors, ACS Nano 8 (2014) 7746-7754.

[22] Y. Zhang, J. Yu, H. N. Bomba, Y. Zhu, Z. Gu, Mechanical force-triggered drug delivery, Chem. Rev. 116 (2016) 12536-12563.

[23] T. O. Tasci, D. Disharoon, R. M. Schoeman, K. Rana, P. S. Herson, D.W. M. Marr, K. B. Neeves, Enhanced fibrinolysis with magnetically powered colloidal microwheels, Small, 13 (2017) 1700954.

[24] H. Du, C. Li, Y. Luan, Q. Liu, W. Yang, Q. Yu, D. Li, J. L. Brash, H. Chen, An antithrombotic hydrogel with thrombinresponsive fibrinolytic activity: breaking down the clot as it forms, Mater. Horiz. 3 (2016) 556-562.

[25] S. T. Gunawan, K. Kempe, T. Bonnard, J. Cui, K. Alt, L. S. Law, X. Wang, E. Westein, G. K. Such, K. Peter, C. E. Hagemeyer, F. Caruso, Multifunctional thrombin- activatable 
polymer capsules for specific targeting to activated platelets, Adv. Mater. 27 (2015) $5153-5157$.

[26] J. N. Marsh, A. Senpan, G. Hu, M. J. Scott, P. J. Gaffney, S. A. Wickline, G.M. Lanza, Fibrin-targeted perfluorocarbon nanoparticles for targeted thrombolysis, Nanomedicine 2 (2007) 533-543.

[27] J. Y. Kim, J. H. Ryu, D. Schellingerhout, I. C. Sun, S. K. Lee, S. Jeon, J. Kim, I. C. Kwon, M. Nahrendorf, C. H. Ahn, K. Kim, D. E. Kim, Direct imaging of cerebral thromboemboli using computed tomography and fibrin-targeted gold nanoparticles, Theranostics 5 (2015) 1098-1114.

[28] G. Fredman, N. Kamaly, S. Spolitu, J. Milton, D. Ghorpade, R. Chiasson, G. Kuriakose, M. Perretti, O. Farokzhad, I. Tabas, Targeted nanoparticles containing the proresolving peptide Ac2-26 protect against advanced atherosclerosis in hypercholesterolemic mice, Sci. Transl. Med. 7 (2015) 275ra20.

[29] G. Lippi, C. Mattiuzzi, E. J. Favaloro, Novel and emerging therapies: thrombus-targeted fibrinolysis, Semin. Thromb. Hemost. 39 (2013) 048-058.

[30] M. Juenet, R. Aid-Launais, B. Li, A. Berger, J. Aerts, V. Ollivier, A. Nicoletti, D. Letourneur, C. Chauvierre, Thrombolytic therapy based on fucoidan-functionalized polymer nanoparticles targeting P-selectin, Biomaterials 156 (2018) 204-216.

[31] P. A. Gurbel, C. M. O’Connor, M. R. Dalesandro, V. L. Serebruany, Relation of soluble and platelet P-selectin to early outcome in patients with acute myocardial infarction after thrombolytic therapy, Am. J. Cardiol. 87 (2001) 774-777. 
[32] B. Li, M. Juenet, R. Aid-Launais, M. Maire, V. Ollivier, D. Letourneur, C. Chauvierre, Development of polymer microcapsules functionalized with fucoidan to target p-selectin overexpressed in cardiovascular diseases, Adv. Healthc. Mater. 6 (2017) 1601200.

[33] J. Zhou, D. Guo, Y. Zhang, W. Wu, H. Ran, Z. Wang, Construction and evaluation of $\mathrm{Fe}_{3} \mathrm{O}_{4}$-based PLGA nanoparticles carrying rtPA used in the detection of thrombosis and in targeted thrombolysis, ACS Appl. Mater. Inter. 6 (2014) 5566-5576.

[34] D. E. Barre, Arginyl-glycyl-aspartyl (RGD) epitope of human apolipoprotein (a) inhibits platelet aggregation by antagonizing the IIb subunit of the fibrinogen (GPIIb/IIIa) receptor. Thromb. Res. 119 (2007) 601-607.

[35] H. F. Langer, M. Gawaz, Platelet-vessel wall interactions in atherosclerotic disease, Thromb. Haemost. 99 (2008) 480-486.

[36] H. D. Shah, R. K. Goyal, Glycoprotein IIb/IIIa receptor and its inhibition: a platelet-directed therapeutic strategy, Ind. J. Pharmacol. 36 (2004) 133-139.

[37] Y. Z. Xiang, L.Y. Kang, X. M. Gao, H. C. Shang, J. H. Zhang, B. L. Zhang, Strategies for antiplatelet targets and agents, Thromb. Res. 123 (2008) 35-49.

[38] J. Sánchez-Cortés, M. Mrksich, The platelet integrin $\alpha_{\mathrm{IIb}} \beta_{3}$ binds to the RGD and AGD motifs in fibrinogen, Chem. Biol. 16 (2009) 990-1000.

[39] R. Srinivasasn, R. E. Marchant, A. S. Gupta, In vitro and in vivo platelet targeting by cyclic rgd-modified liposomes, J. Biomed. Mater. Res. A. 93 (2010) 1004-1015.

[40] S. Liu, X. Feng, R. Jin, G. Li, Tissue plasminogen activator-based nanothrombolysis for ischemic stroke, Expert Opin. Drug Deliv. 15 (2018) 173-184. 
[41] S. Absar, K. Nahar, Y. M. Kwon, F. Ahsan, Thrombus-targeted nanocarrier attenuates bleeding complications associated with conventional thrombolytic therapy, Pharm. Res. 30 (2013) 1663-1676.

[42] S. Chen, S. Wang, M. Kopytynski, M. Bachelet, R. Chen, Membrane-anchoring, comb-like pseudopeptides for efficient, pH-mediated membrane destabilization and intracellular delivery, ACS Appl. Mater. Interfaces 9 (2017) 8021-8029.

[43] J. Connor, M. B. Yatvin, L. Huang, pH-sensitive liposomes: acid-induced liposome fusion, Proc. Natl. Acad. Sci. U S A. 81 (1984) 1715-1718.

[44] N. Duzgunes, S. Nir, Mechanisms and kinetics of liposome cell interactions, Adv. Drug Delivery Rev. 40 (1999) 3-18

[45] J. M. White, Membrane Fusion, Science 258 (1992) 917-924.

[46] D. K. Struck, D. Hoekstra, R. E. Pagano, Use of resonance energy transfer to monitor membrane fusion, Biochemistry 20 (1981) 4093-4099.

[47] J. T. Kleinman, A. E. Hillis, L. C. Jordan, ABC/2: estimating intracerebral haemorrhage volume and total brain volume and predicting outcome in children, Dev. Med. Child. Neurol. 53 (2011) 281-284.

[48] S. Chen, R. Chen, A virus-mimicking, endosomolytic liposomal system for efficient, ph-triggered intracellular drug delivery, ACS Appl. Mater. Inter. 8 (2016) 22457-22467. 
[49] F. Atyabi, A. Farkhondehfai, F. Esmaeili, R. Dinarvand, Preparation of pegylated nano-liposomal formulation containing SN-38: in vitro characterization and in vivo biodistribution in mice. Acta Pharm. 59 (2009) 133-44.

[50] R. Gref, A. Domb, P. Quellec, T. Blunk, R. H. Müller, J. M. Verbavatz, R. Langer, The controlled intravenous delivery of drugs using PEG-coated sterically stabilized nanospheres, Adv. Drug Delivery Rev. 64 (2012) 316-326.

[51] S. Koudelka, R. Mikulik, J. Mašek, M. Raška, P. T. Knotigová, A. D. Miller, J. Turánek, Liposomal nanocarriers for plasminogen activators, J. Control. Release 227 (2016) 45-57.

[52] D. R. Elias, A. Poloukhtine, V. Popik, A. Tsourkas, Eff ect of ligand density, receptor density, and nanoparticle size on cell targeting, Nanomedicine 9 (2013) 194-201.

[53] R. J. Y. Ho, H. P. Ting-Beall, B. T. Rouse, L. Huang, Kinetic and ultrastructural studies of interactions of target-sensitive immunoliposomes with herpes simplex virus, Biochemistry 27 (1988) 500-506.

[54] R. J. Y. Ho, B. T. Rouse, L. Huang, Target-sensitive immunoliposomes: preparation and characterization, Biochemistry 25 (1986) 5500-5506.

[55] J. F. Liang, H. Song, Y. T. Li, V. C. Yang, A novel heparin/protamine-based pro-drug type delivery system for protease drugs, J. Pharm. Sci. 89 (2000) 664-673.

[56] Y. J. Park, J. Liang, Z. Yang, V. C. Yang, Controlled release of clot-dissolving tissue-type plasminogen activator from a poly(L-glutamic acid) semi-interpenetrating polymer network hydrogel, J. Control. Release 75 (2001) 37-44.

[57] R. Pannell, S. Li, V. Gurewich, Highly effective fibrinolysis by a sequential synergistic combination of mini-dose tPA plus low-dose mutant proUK, PLoS One 10 (2015) e0122018. 
[58] R. Aisina, L. Mukhametova, S. Varfolomevev, Synergistic fibrinolysis: The combined effects of tissue plasminogen activator and recombinant staphylokinase in vitro, Biochim. Biophys. Acta 1860 (2016) 629-635. 

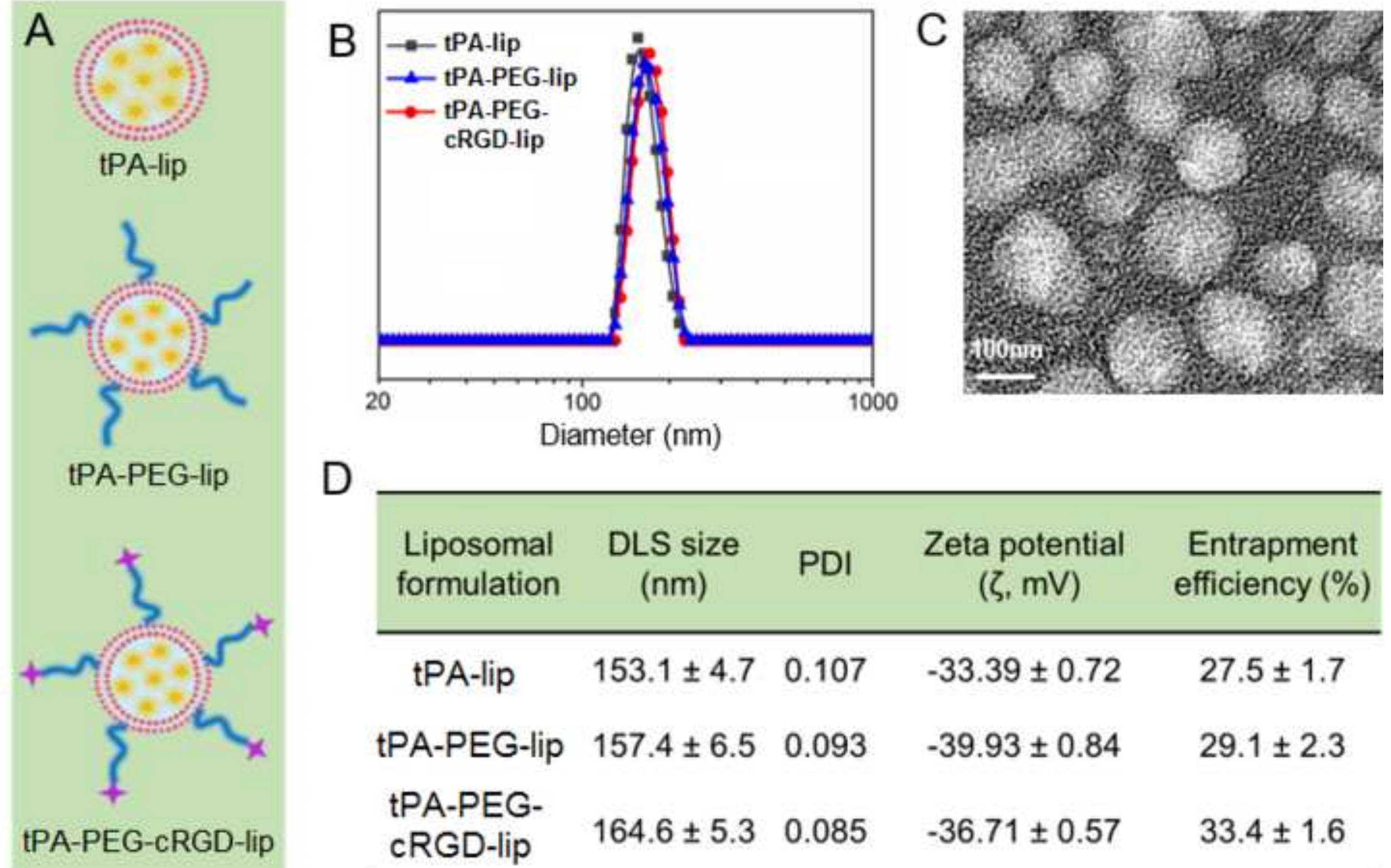

D

\begin{tabular}{ccccc}
\hline $\begin{array}{c}\text { Liposomal } \\
\text { formulation }\end{array}$ & $\begin{array}{c}\text { DLS size } \\
(\mathrm{nm})\end{array}$ & PDI & $\begin{array}{c}\text { Zeta potential } \\
(\zeta, \mathrm{mV})\end{array}$ & $\begin{array}{c}\text { Entrapment } \\
\text { efficiency }(\%)\end{array}$ \\
\hline tPA-lip & $153.1 \pm 4.7$ & 0.107 & $-33.39 \pm 0.72$ & $27.5 \pm 1.7$ \\
$\begin{array}{l}\text { tPA-PEG-lip } \\
\begin{array}{l}\text { tPA-PEG- } \\
\text { cRGD-lip }\end{array}\end{array}$ & $164.4 \pm 6.5$ & 0.093 & $-39.93 \pm 0.84$ & $29.1 \pm 2.3$ \\
\hline
\end{tabular}



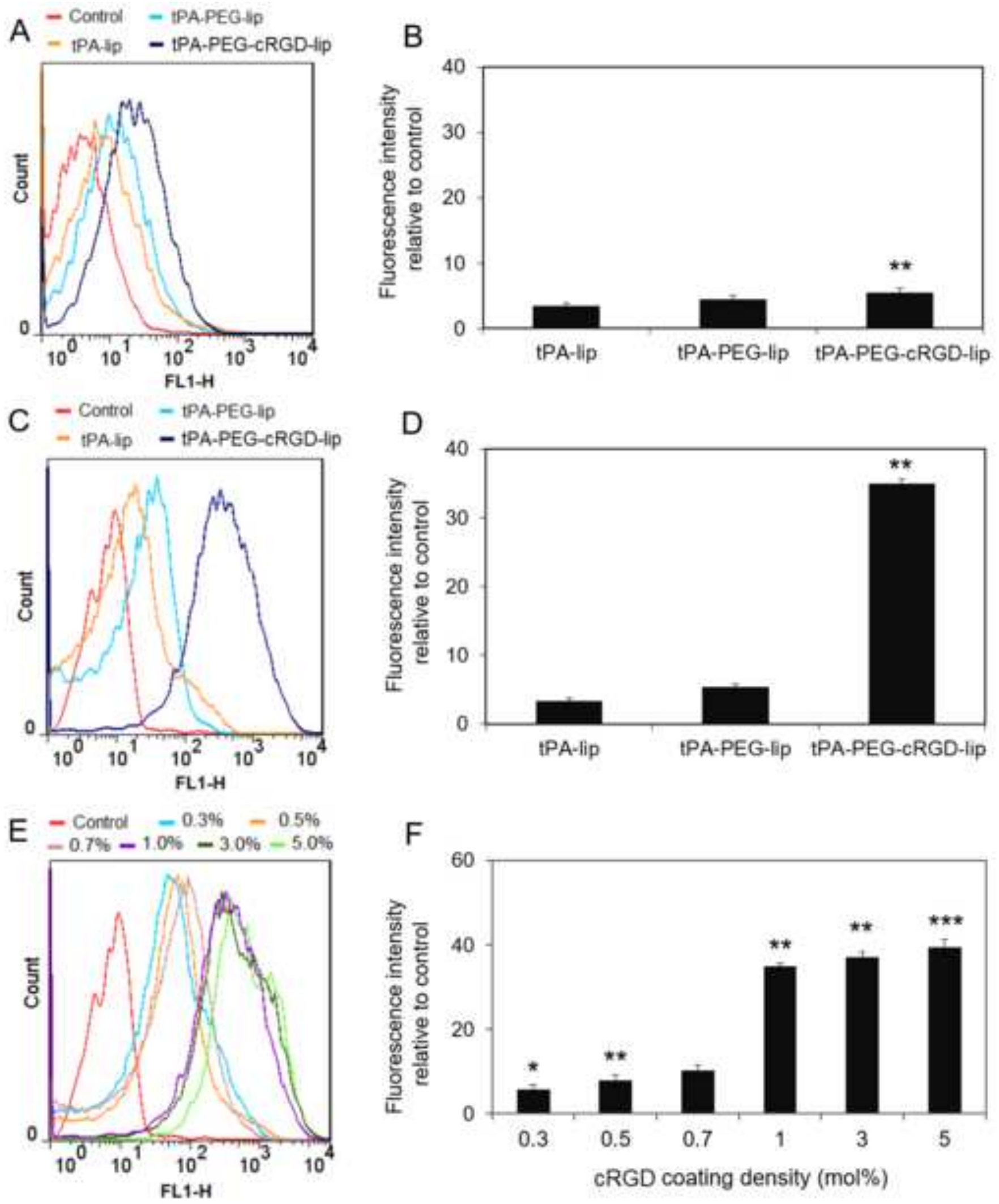
Click here to download high resolution image

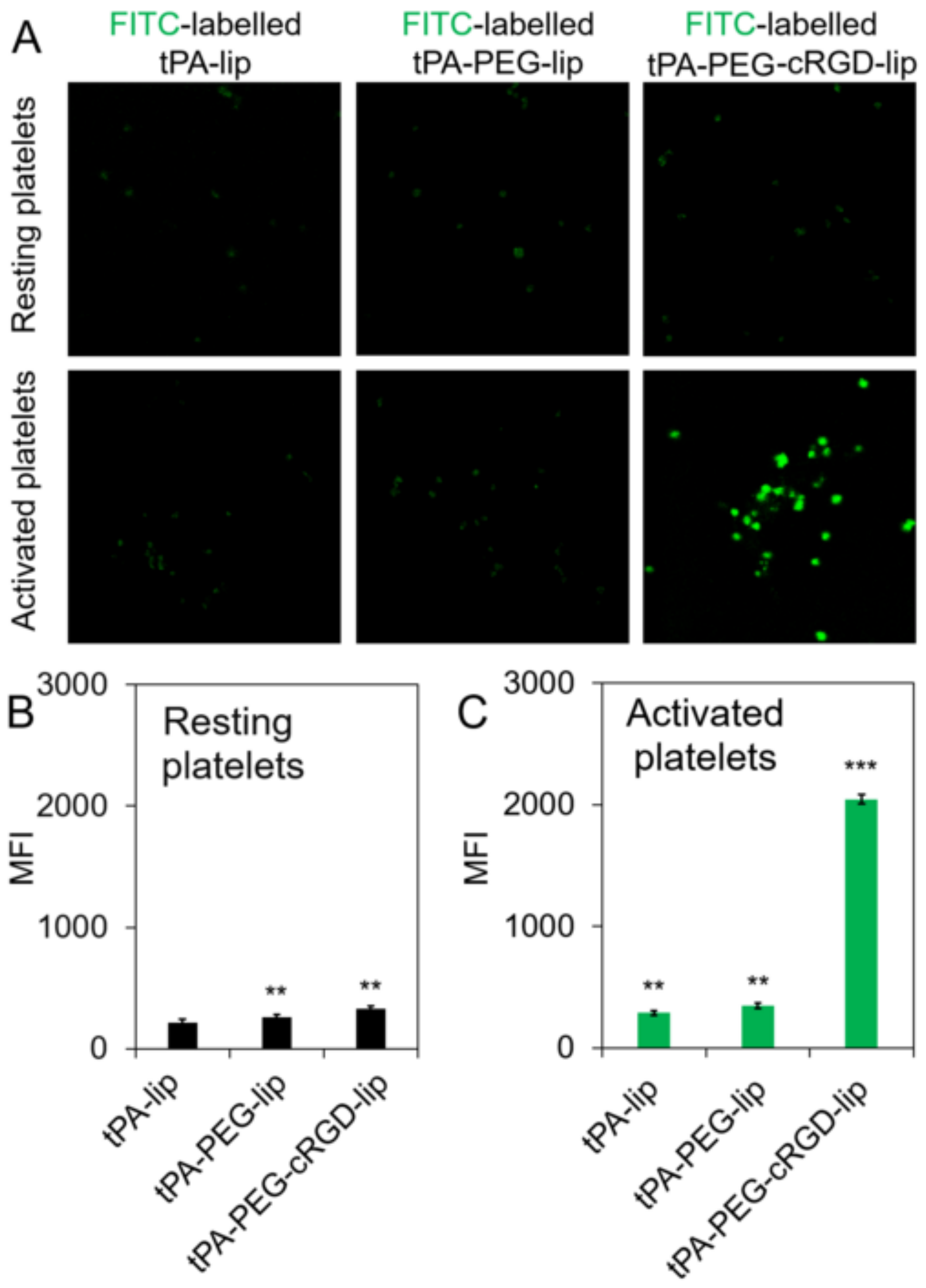


A

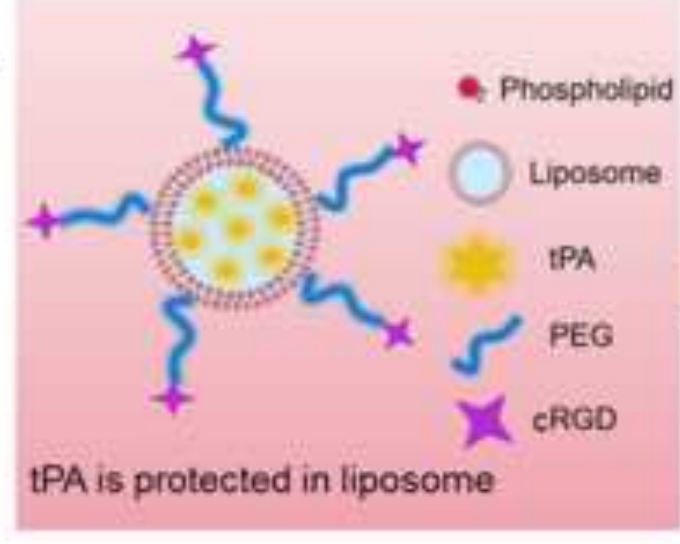

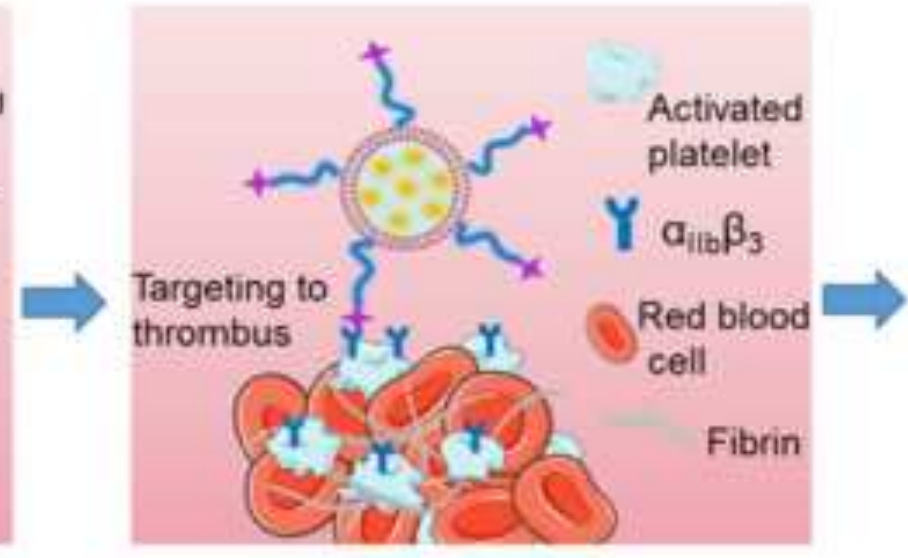

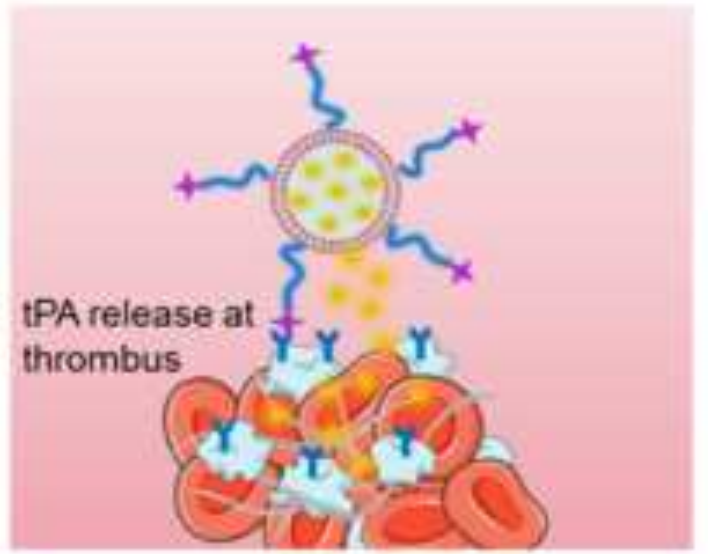

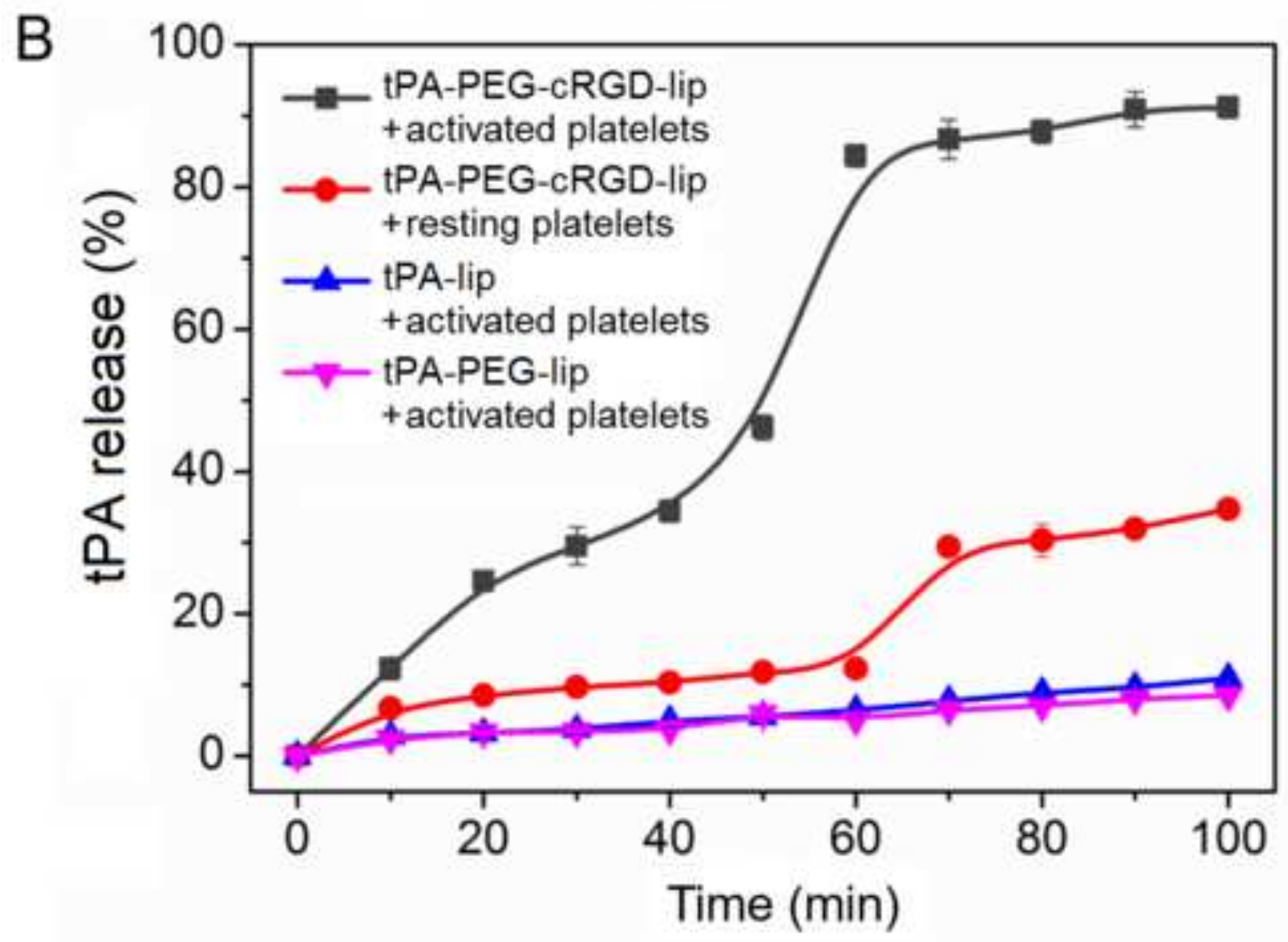




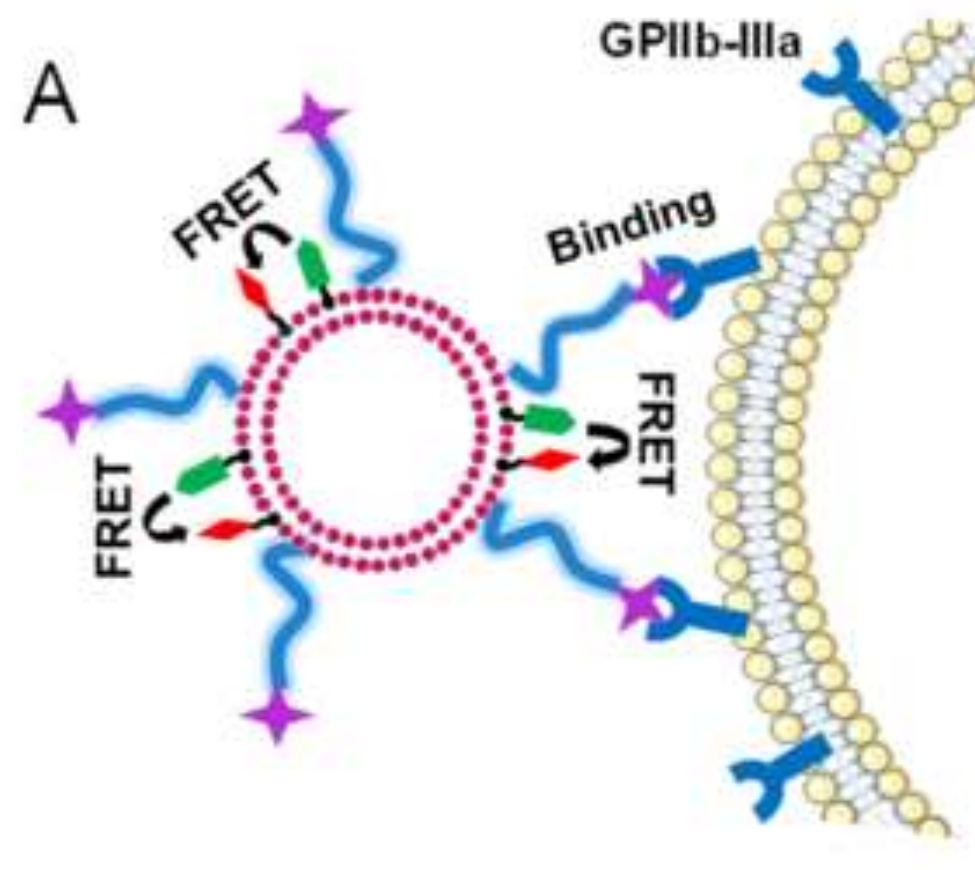

Activated platelet
membrane

B

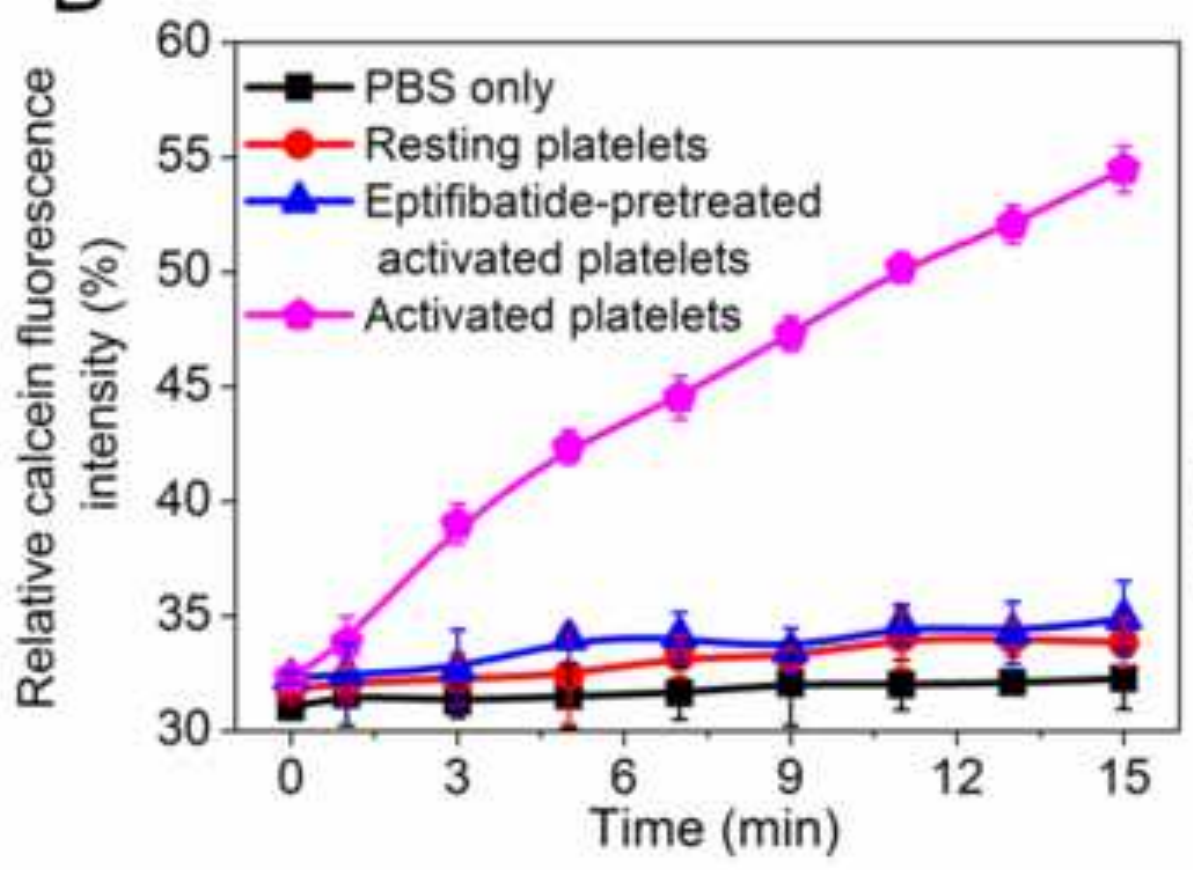

\section{Membrane fusion}
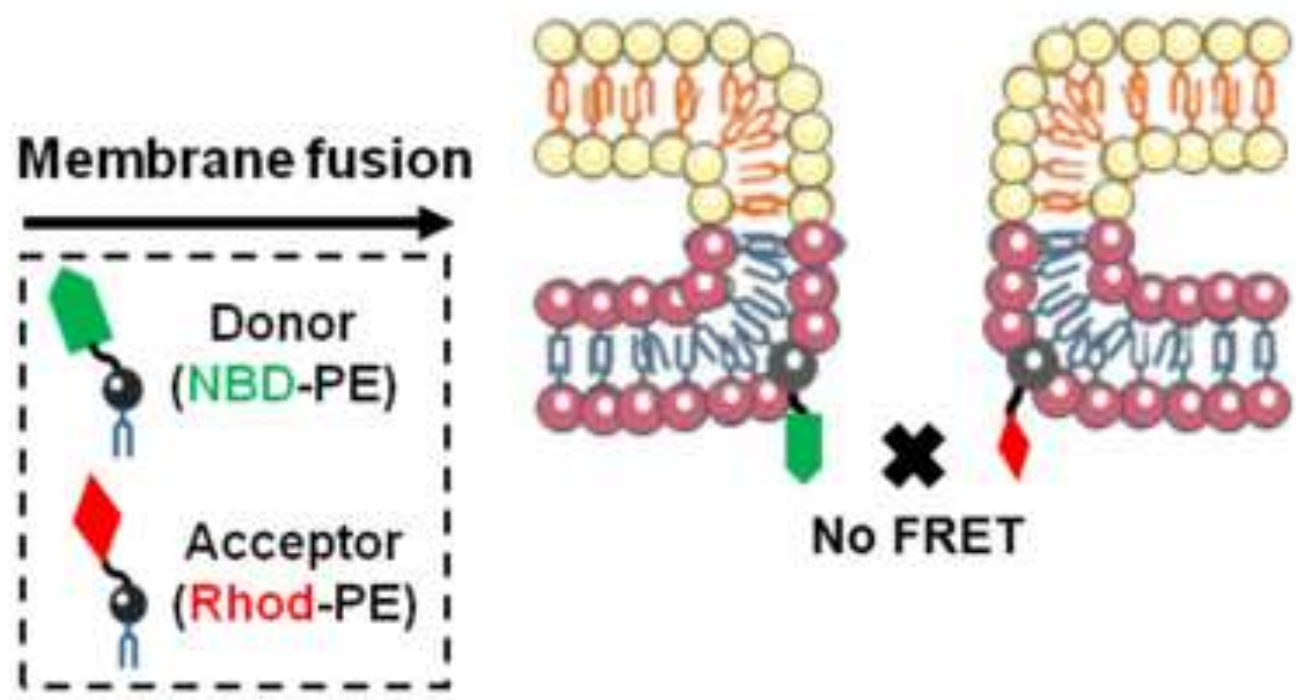

No FRET

C

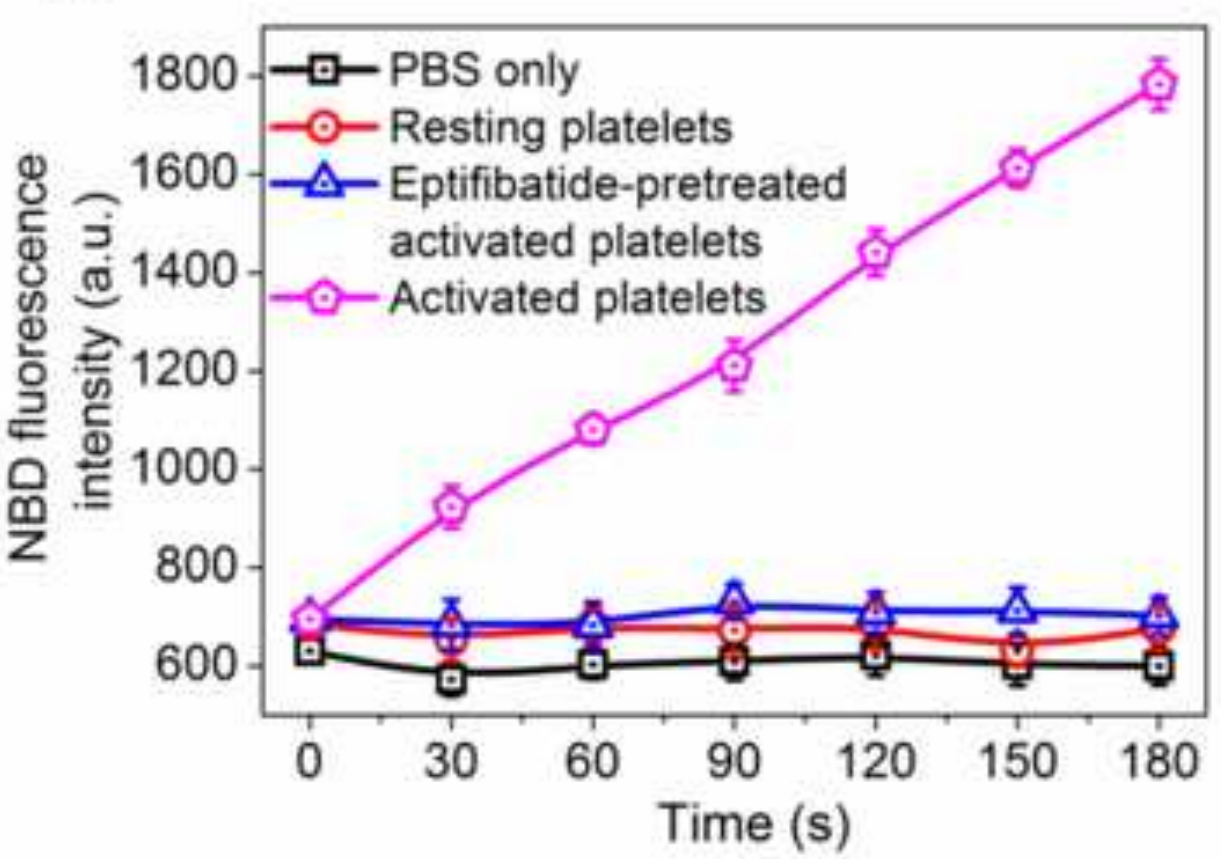


A
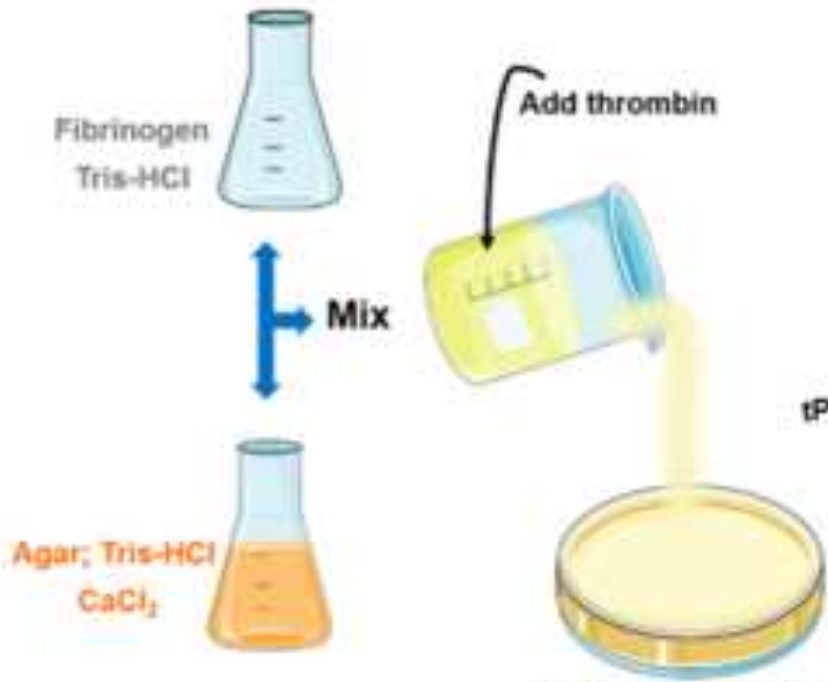

Make wells

Add drugs

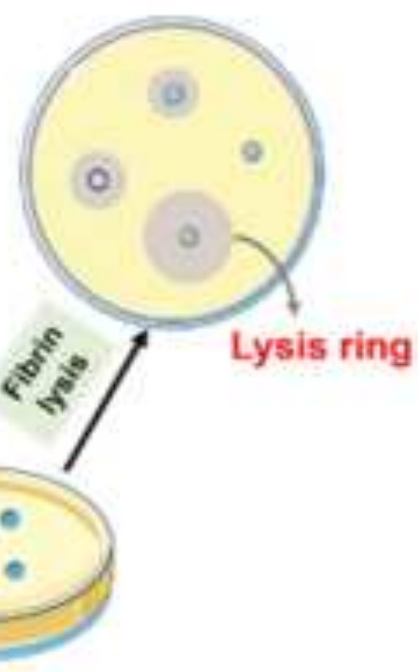

Fibrin clot model

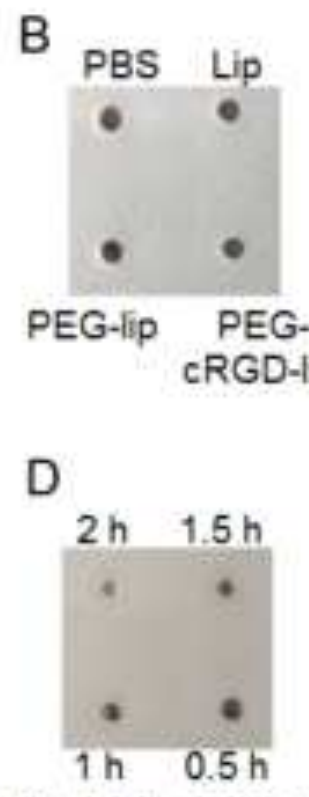

tPA-PEG-cRGD-lip

+ Resting platelets

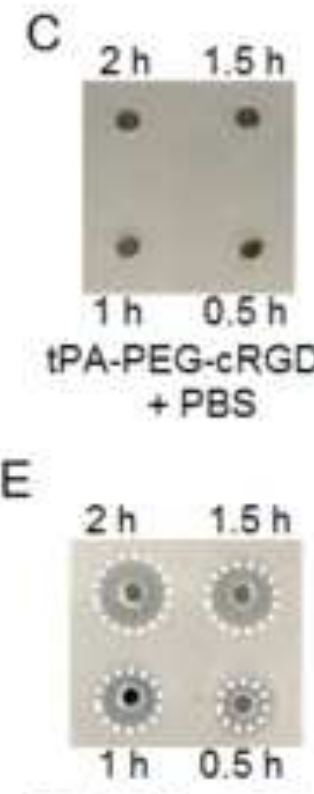

tPA-PEG-cRGD-lip

+ Activated paltelets

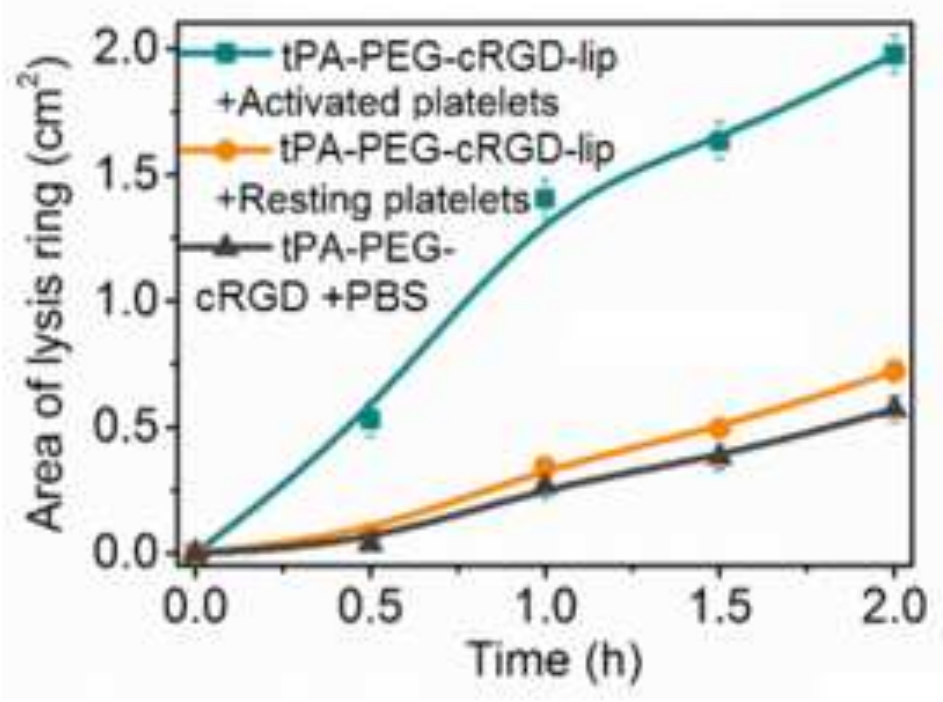

G

$\mathrm{H}$
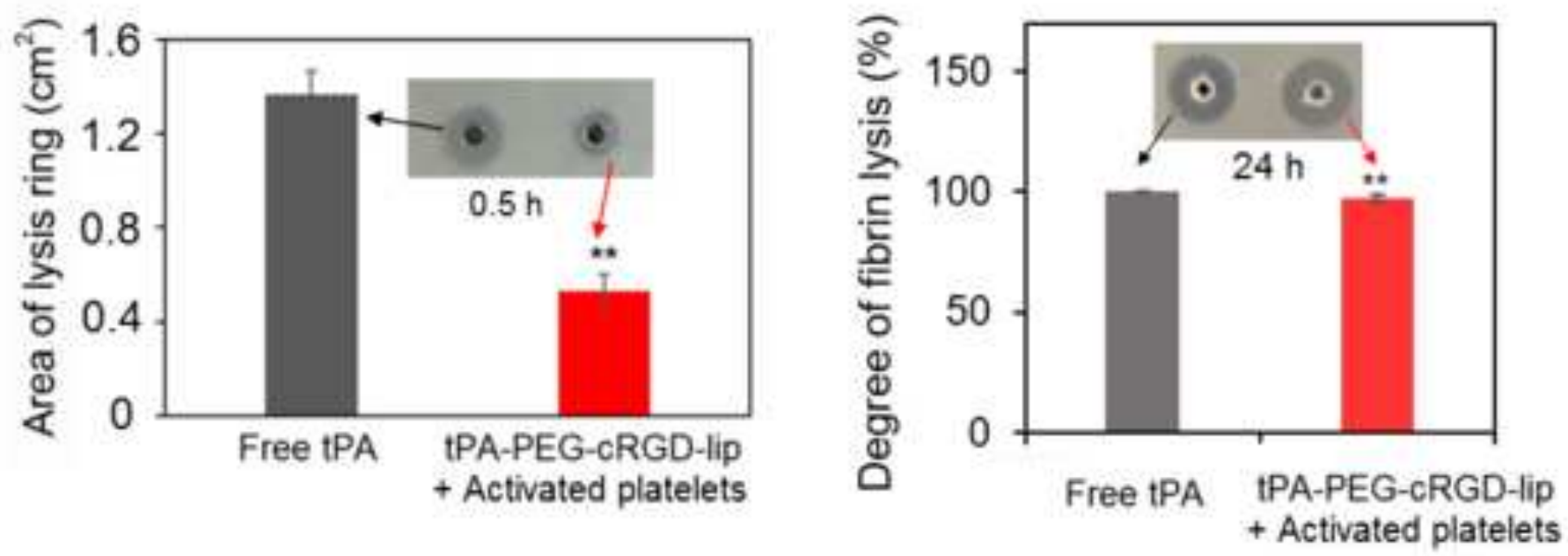
Figure 7

Click here to download high resolution image
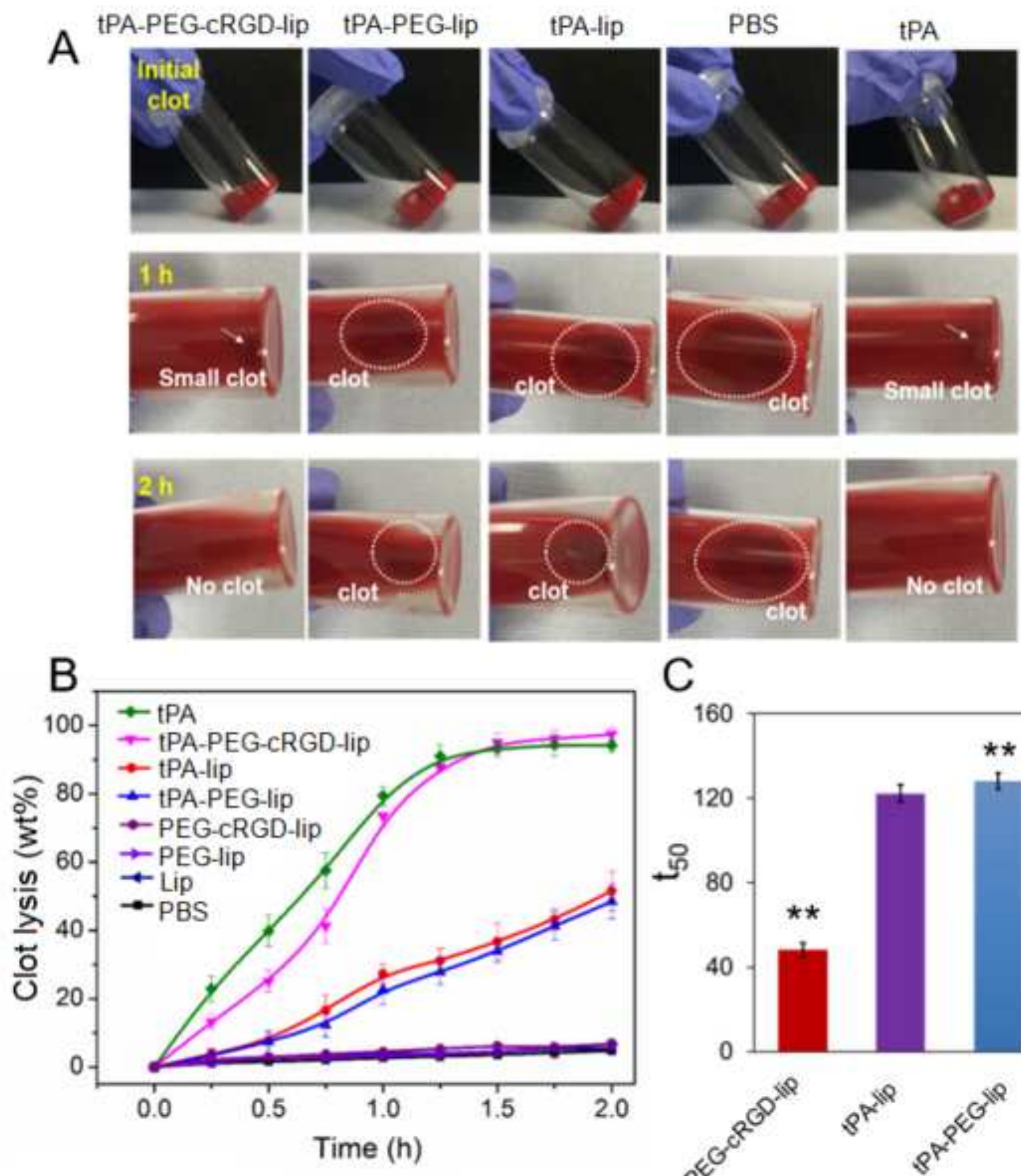

C

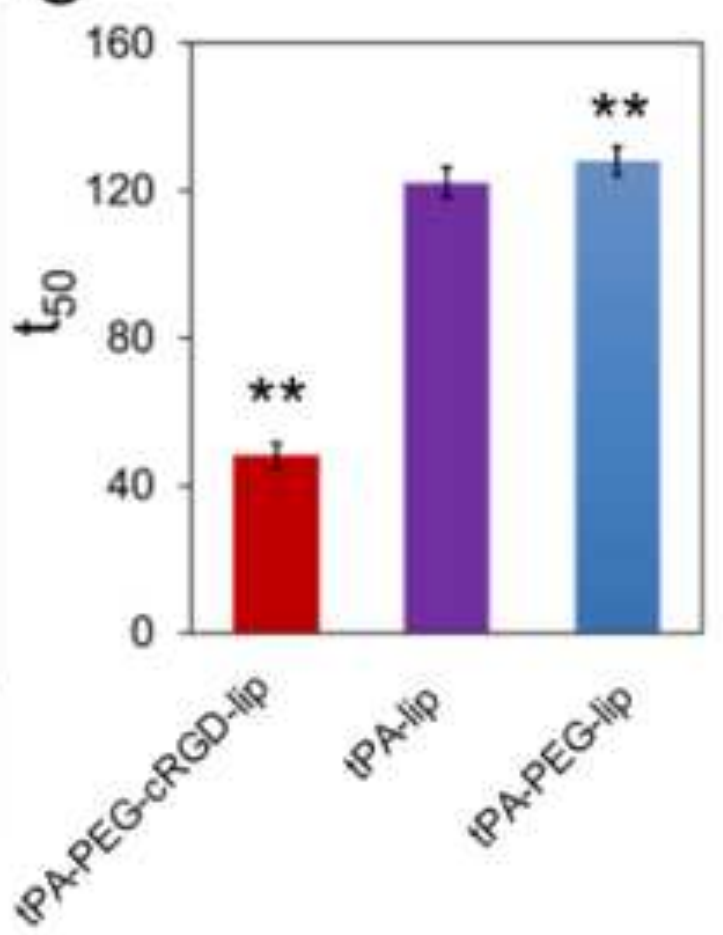


Highlights
Click here to download Supplementary Material: Highlights.docx Click here to download Supplementary Material: Highights.docx (n)

(1)

(1)

(1) (1) (1) (1) (1)

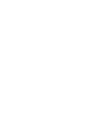
(1)

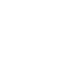

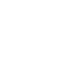
(1)

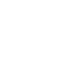
(1)

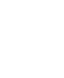
.

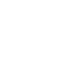

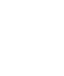
. . 
Supplementary Data
Click here to download Supplementary Material: Revised Supplementary Data.doc)

Click here to download Supplementary Material: Revised Supplementary Data.docx

ck here to download Supplementary Material: Revised Supplementary Data.docx

(1)

(n)

(1)

(1)

(1)

(1)

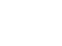

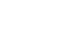

.

.

.

.

.

.

.

.

.

.

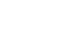

.

.

.

.

.

.

.

.

.

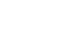

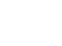

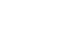

